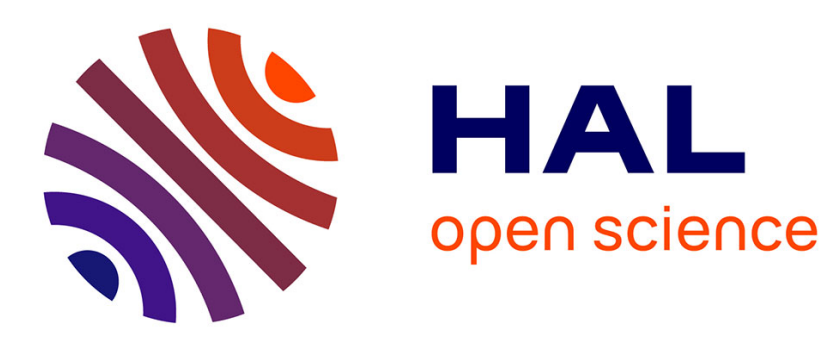

\title{
Dynamic practical stabilization of sampled-data linear distributed parameter systems
}

\author{
Ying Tan, Emmanuel Trélat, Yacine Chitour, Dragan Nesic
}

\section{To cite this version:}

Ying Tan, Emmanuel Trélat, Yacine Chitour, Dragan Nesic. Dynamic practical stabilization of sampled-data linear distributed parameter systems. 48h IEEE Conference on Decision and Control (CDC), Dec 2009, Shanghai, China. 10.1109/cdc.2009.5399931 . hal-00366232v2

\section{HAL Id: hal-00366232 \\ https://hal.science/hal-00366232v2}

Submitted on 19 Sep 2009

HAL is a multi-disciplinary open access archive for the deposit and dissemination of scientific research documents, whether they are published or not. The documents may come from teaching and research institutions in France or abroad, or from public or private research centers.
L'archive ouverte pluridisciplinaire HAL, est destinée au dépôt et à la diffusion de documents scientifiques de niveau recherche, publiés ou non, émanant des établissements d'enseignement et de recherche français ou étrangers, des laboratoires publics ou privés. 


\title{
DYNAMIC PRACTICAL STABILIZATION OF SAMPLED-DATA LINEAR DISTRIBUTED PARAMETER SYSTEMS*
}

\author{
YING TAN ${ }^{\dagger}$, EMMANUEL TRÉLAT $\ddagger$, YACINE CHITOUR §, AND DRAGAN NEŠIĆ $₫$
}

\begin{abstract}
In this paper, dynamic practical stability properties of infinite-dimensional sampleddata systems are discussed. A family of finite-dimensional discrete-time controllers are first designed to uniformly exponentially stabilize numerical approximate models that are obtained from space and time discretizations. Sufficient conditions are provided to ensure that these controllers can be used to drive trajectories of infinite-dimensional sampled-data systems to a neighborhood of the origin by properly tuning the sampling period, the space and time discretization parameters and choosing an appropriate filtering process for initial conditions.
\end{abstract} tions.

Key words. Distributed systems, Practical stability, Sampled-data, Space and time discretiza-

AMS subject classifications. 68M14, 93C57

1. Introduction. Linear distributed parameter systems (LDPS) arise in a range of different processes such as optical telecommunications, fluid flows, thermal processes, biology, chemistry, environmental sciences, mechanical systems, and so on. LDPS are modelled by linear partial differential equations (PDEs) or abstract differential equations in an infinite-dimensional space, as opposed to linear lumped parameter systems (LLPS) that are modelled by linear ordinary differential equations (ODEs) in a finite-dimensional space.

In this paper, we consider systems governed by partial differential equations with appropriate initial and boundary conditions that can be represented by the following abstract differential equation,

$$
\dot{x}(t)=A x(t)+B u(t), x(0)=x_{0} \in X, \forall t \geq 0 .
$$

Here, the state $x(t)$ belongs to a Banach space $X$ and the control input $u(t)$ belongs to a subset of a Banach space $U$. The operator $A$ maps from $D(A)$ to $X, D(A)$ is the domain of $A$, which is a subset in $X$. The operator $B$ is a control operator (in general, unbounded) on $U$.

Nowadays, most control systems are implemented using digital technology since it is very cheap, fast, relatively easy to operate, flexible and reliable. This motivates the investigation of the so called sampled-data systems that consist of a continuous-time plant or process controlled by a discrete-time controller, as discussed in [5, 30, 31]. The plant and the controller are interconnected via the analog-to-digital (A-D) and digital-to-analog (D-A) converters. Consequently, the designed controller needs to be time-discretized in order to be implemented using the digital technology. Due to

${ }^{*}$ This work was supported by the Australian Research Council (ARC) Discovery Project (DP0664501).

$\dagger$ The Department of Electrical and Electronic Engineering, The University of Melbourne, Melbourne, VIC 3010, Australia. (y.tan@ee.unimelb.edu.au).

¥Université d'Orléans, Laboratoire MAPMO CNRS, UMR 6628 Fédération Denis Poisson, FR 2964 Bâtiment de Mathématiques BP 6759, 45067 Orléans cedex 2, France. (emmanuel.trelat@univ-orleans.fr).

$\S$ Laboratoire des signaux et systèmes, Université Paris-Sud, CNRS, Supélec, 91192 Gif-SurYvette, France (yacine.chitour@lss.supelec.fr)

I The Department of Electrical and Electronic Engineering, The University of Melbourne, Melbourne, VIC 3010, Australia. (d.nesic@ee.unimelb.edu.au). 
prevalence of the computer controlled systems, it is often assumed that System (1.1) is between a sampler and a zero-order-hold. Let $T>0$ denote a sampling period. The control signal is assumed to be piecewise constant,

$$
u(t)=u(k T) \triangleq u(k), \forall t \in[k T,(k+1) T), k \in \mathbb{N},
$$

where $\mathbb{N}$ is the set of integers. In the sequel, the following "sampled-data system" is obtained.

$$
\dot{x}(t)=A x(t)+B u(k T), \forall t \in[k T,(k+1) T), k \in \mathbb{N} .
$$

The control input $u(k T)$ needs to be designed so that trajectories of the sampled-data system (1.3) converge to the origin, or a neighborhood of the origin.

Sampled-data control of linear infinite-dimensional system (1.1) has been discussed in $[4,9,14,16,18,19,28,29,34,35,37]$, and references cited therein. In most of these references, an infinite-dimensional continuous-time feedback controller was first designed to stabilize the system (1.1) without consideration of sampling in time, followed by a time discretization in order to implement digitally. This is an "indirect method", which consists of designing a controller on the continuous model, and then in discretizing the closed-loop system.

Given a controlled PDE (1.1), it is however not always possible to guess an expression of a feedback controller stabilizing the system. We propose a direct approach, which consists in designing such a controller from finite-dimensional approximations that are obtained from space and time discretization. The reason for doing so lies in several aspects. First of all, in general, analytical solutions of the infinite-dimensional system (1.1) or (1.3) are not possible to explicitly characterize. In engineering applications, it is natural to use numerical solutions generated by numerical approximate models, themselves arising from numerical algorithms such as finite difference methods, finite element methods, Galerkin approximations and so on. As there are many numerical algorithms available in literature, engineers just need to pick up one suited for the particular application. Furthermore, it can be efficient for some applications when accurate discrete models are available. Secondly, it is appealing for engineers to design controllers for discretization models. Although there is a large number of publications on stabilization of systems like (1.1), see Russell [38], Lions [26], Komornik [20], Curtain and Zwart [11], Lasiecka and Triggiani [24] and references therein, it may be rather difficult to find control laws for some infinite-dimensional system in the form of (1.1). At last, the family of finite-dimensional discrete-time controllers are easy to be implemented. Indeed, while infinite-dimensional controllers are theoretically relevant, the effective controller has to be finite-dimensional in order to be implemented digitally.

It is by now well-known that the scheme "control design/ discretization" is not commutative (see, for example, [44]). Whereas it is quite easy to prove convergence results for an indirect method, with standard assumptions and a standard Lax procedure (see [25]), obtaining a convergence result for a direct approach may be really challenging, due to a possible loss of uniformity. Actually, when implementing a direct approach, the standard assumptions which are usually ensuring the convergence of a given scheme, namely, consistency plus uniform boundedness (or stability), are not enough in general to ensure the convergence of the family of control inputs designed from the approximate models towards the control input of the continuous-time model. As explained in [44], this phenomenon is due to an interference of high frequencies 
with the mesh of the discretization; this interference may create spurious high frequency oscillations which, as in a resonance phenomenon, leads to the divergence of the direct procedure.

In this paper, we assume that there exist a family of finite-dimensional discretetime approximate models in the vector spaces $X_{h}$ and $U_{h}$ after space and time discretization. Here $h$ is a parameter for the space approximation and $\Delta t$ is a parameter for time discretization. Both $h$ and $\Delta t$ are sufficiently small. This family of approximate models, represented in terms of $x_{h}^{a}(j \Delta t)^{1}$, take the following form,

$$
x_{h}^{a}((j+1) \Delta t)=A_{h, \Delta t}^{a} x_{h}^{a}(j \Delta t)+B_{h, \Delta t}^{a} u_{h, \Delta t}(j \Delta t),
$$

with the initial condition $x_{h}^{a}(0) \in X_{h}$ and $j \in \mathbb{N}$. Here $x_{h}^{a} \in X_{h}, u_{h} \in U_{h}$ and $A_{h, \Delta t}^{a}: X_{h} \rightarrow X_{h}$ and $B_{h, \Delta t}^{a}: U_{h} \rightarrow X_{h}$.

Furthermore, we assume that the family of controllers $u_{h, \Delta t}$ are carefully designed in the sense that they can uniformly exponentially stabilize approximate models (1.4). More specifically, the solutions of the approximate models (1.4) satisfy

$$
\left\|x_{h}^{a}((j+1) \Delta t)\right\|_{X_{h}} \leq M_{a} e^{-\lambda_{a} j \Delta t}\left\|x_{h}^{a}(0)\right\|_{X_{h}},
$$

for some positive constants $M_{a}$ and $\lambda_{a}$ that are independent of the choice $h$ and $\Delta t$.

As mentioned in [33], it is not always possible to construct a proper control sequence $u_{h, \Delta t}$ to uniformly exponentially stabilize a family of finite-dimensional approximations (1.4) due to the existence of spurious high frequency modes. Uniform stability properties (controllability and/or observability) of a family of approximation control systems have been investigated in $[12,33,41,42,43,44]$ for different discretization processes, on different systems. In this paper, for simplicity of the presentation, we just assume the existence of such "good" controllers. How to design them is outside the scope of this paper. We however refer readers to $[1,3,13,17,24]$ and references therein as concerns results regarding the design of control laws having such uniform properties, based on a Riccati procedure.

Once a "good" control sequence $u_{h, \Delta t}$ is available, our aim is to find (sharp) sufficient conditions ensuring that this control sequence can be used to drive trajectories of the infinite-dimensional sampled-data system (1.3) to the origin (or a small neighborhood of the origin).

Note that the control input applied to the sampled-data system (1.3) is computed from approximate models (1.4). In other words, the controller $u(k T)$ in (1.3) is generated from a family of finite-dimensional discrete-time controllers $u_{h, \Delta t}(j \Delta t)$. Thus $u(k T)$ is not in a typical state-feedback form that is obtained from state measurement $x(t)$ of the system (1.1). As $u_{h, \Delta t}(j \Delta t)$ can be treated as a kind of "memory" variable, by adopting the terminology introduced in [39, 6], "dynamic (practical) stabilization" is used in this paper.

It is worthwhile to highlight that we do not assume the existence of an infinitedimensional controller that can practically exponentially stabilize the exact infinitedimensional continuous-time system (1.1) nor prove that finite-dimensional discretetime controllers computed from numerical approximations converge uniformly to the desired one as the discretization parameters tend to zero as done in the literature, in particular, in the context of the Riccati theory, see, for example, $[1,3,13,17$, $22,24,27]$ and references therein. Our result (Theorem 3.3) only provides sufficient

\footnotetext{
${ }^{1}$ In this paper, $x_{h}^{a}(\cdot)$ represents trajectories generated from finite-dimensional discrete-time approximate models in the vector space $X_{h}$.
} 
conditions to ensure the "practical stability properties" of a general class of sampleddata LDPS by using a dynamic feedback that can "uniformly exponentially stabilize" numerical approximate models (1.4). More precisely, we obtain sufficient conditions gauranteeing that for any given positive pair $(\Delta, \nu)$, there exists a filtering process depending on $(\Delta, \nu)$ such that, for any filtered initial condition, trajectories of the infinite-dimensional sampled-data system (1.3), with the control input sequence $u(k T)$ generated from $u_{h, \Delta}(j \Delta t)$, will converge to a $\nu$-neighborhood of origin by properly tuning the sampling period $T$ and the numerical discretization parameters $(h$ and $\Delta t)$ and choosing an appropriate filtering process. These sufficient conditions are listed below.

1 The trajectories of the numerical approximations (1.4) have to be "good" enough to well approximate the trajectories of the exact system (1.1);

2 the control sequence $u_{h, \Delta t}$ has to be uniformly bounded with respect to space and time discretization parameters;

3 the filtering process determined by $(\Delta, \nu)$ is required in order to filter out high frequency components of the initial data;

4 in addition, the filtering process must be compatible with the uniform stability properties of the approximate models.

To the best of our knowledge, it is the first time that the issue of practical exponential stability properties of a general class of sampled-data LDPS is addressed by using a dynamic feedback generated by numerical approximate models (1.4). Our result can provide useful guidelines to choose the sampling period, appropriate numerical schemes including discretization parameters as well as the proper filtering process. It is also worthwhile to highlight that, although conditions in the main result stated below are only sufficient, they are "sharp" in the following sense: if one of the above mentioned conditions does not hold true, then the conclusion of the main results may fail (counterexamples can be found in literature).

The present paper is organized as follows. Section 2 provides necessary preliminaries as well as the problem formulation. Sufficient conditions and the main results are stated and discussed in Section 3. Proofs of the main results are provided in Section 4 and then they are followed by the conclusions in Section 5 .

2. Preliminaries and Problem Formulation. In this paper, $X$ and $U$ are Banach spaces with their norms denoted as $\|\cdot\|_{X}$ and $\|\cdot\|_{U}$ respectively. The dual space of $X$ is denoted by $X^{\prime}$. Let $S(t)$ denote a strongly continuous semigroup $\left(C_{0^{-}}\right.$ semigroup) on $X$, of generator $\left(A,(D(A))\right.$ in System (1.1). Let $\alpha>0$; then $X_{-\alpha}$ denotes the completion of $X$ for the norm $\|x\|_{-\alpha}=\left\|\left(\beta I_{X}-A\right)^{-\alpha} x\right\|_{X}$, where $\beta \in$ $\rho(A)$ is fixed, $\rho(A)$ being the resolvent set of $A$ and $I_{X}$, the identity in $X$. The semigroup $S(t)$ can be extended to a $C_{0}$-semigroup on $X_{-\alpha}$, denoted by the same symbol, and the generator of this extended semigroup is an extension of $A$, still denoted $A$. With this notation, $A$ is a linear operator from $X$ to $X_{-\alpha}$. Since $A$ generates a $C_{0}$-semigroup, there exists a real number $\omega \in \rho(A)$ such that $A-\omega I_{X}$ is invertible. Let $A^{*}$ be the adjoint of $A$ and $\hat{A}=A-\omega I_{X}$. Then, the fractional powers of $(-\hat{A})^{\alpha}$ are well-defined. $A^{*}$ is the adjoint of $A$. We use $\mathcal{L}(X, Y)$ to denote the space of all linear bounded operators from $X$ to $Y$, where both $X$ and $Y$ are Banach spaces and $\mathcal{L}(X) \triangleq \mathcal{L}(X, X)$ (see, for example [32] for more details about the semi-group and weak solutions of the abstract differential equation (1.1)).

The sets of integers and real numbers are denoted as $\mathbb{N}$ and $\mathbb{R}$ respectively. A continuous function $\gamma: \mathbb{R}_{\geq 0} \rightarrow \mathbb{R}_{\geq 0}$ is said to be of class $\mathcal{K}_{\infty}$ if $\gamma(0)=0, \gamma$ is strictly 
increasing and $\lim _{s \rightarrow \infty} \gamma(s)=\infty$. The set $B_{\Delta}$ is defined as $B_{\Delta} \triangleq\left\{x \in X \mid\|x\|_{X} \leq \Delta\right\}$.

The control operator $B$ in (1.1) is not necessarily bounded. However it is assumed that $B \in \mathcal{L}\left(U, X_{-\alpha}\right)$ and it is admissible (see Definition 2.1).

Definition 2.1. An unbounded linear control operator $B \in \mathcal{L}\left(U, X_{-\alpha}\right)$ is called admissible for the semigroup $S(t)$ if, for every $x_{0} \in X$, the weak solution of (1.1) with $x(0)=x_{0} \in X$ belongs to $X$ for every $t \geq 0$, whenever $u \in \mathcal{L}^{2}([0, \infty], U)$ and (1.1) holds true in $X_{-\alpha}$. The weak solution can be represented in the following form

$$
x(t)=S(t) x_{0}+\int_{0}^{t} S(t-\tau) B u(\tau) d \tau \in X, \forall t \geq 0 .
$$

With the sampled-data controller defined in (1.2), the following weak solutions of the system (1.1) are obtained:

$$
x(t)=S(t-k T) x(k T)+\int_{k T}^{t} S(t-\tau) B u(k T) d \tau,
$$

for all $t \in[k T,(k+1) T)$ and $k \in \mathbb{N}$.

2.1. Numerical approximations. A family of finite-dimensional discrete-time approximations given by (1.4) are obtained by discretizing (1.1) in both time $\Delta t$ and space $h$. In this paper, time discretization is performed after space discretization and the numerical approximations have to be "good" enough to well-approximate the behavior of the system (1.1) (see Assumption 1 and 2 below for a precise definition of "good"). First we introduce adapted assumptions on space approximations, inspired by $[22,24]$. Consider two families $\left(X_{h}\right)_{0<h<h_{0}}$ and $\left(U_{h}\right)_{0<h<h_{0}}$ of finite-dimensional spaces.

Assumption 1. [Consistency of the space semi-discretization scheme] For every $h \in\left(0, h_{0}\right)$, there exist mappings $R_{h}: D\left(\left(-\hat{A}^{*}\right)^{-\alpha}\right)^{\prime} \rightarrow X_{h}, P_{h}: X_{h} \rightarrow D\left(\left(-\hat{A}^{*}\right)^{-\alpha}\right)$, $\bar{R}_{h}: U \rightarrow U_{h}$ and $\bar{P}_{h}: U_{h} \rightarrow U$ such that the following conditions hold.

1 For every $h \in\left(0, h_{0}\right)$, the following holds

$$
R_{h} P_{h}=I_{X_{h}}, \quad \bar{R}_{h} \bar{P}_{h}=I_{U_{h}} .
$$

2 For any $\phi \in D\left(A^{*}\right)$ or any $\psi \in U$, we have

$$
\begin{array}{ll}
\left\|\left\{I_{X}-P_{h} R_{h}\right\} \phi\right\|_{X} \underset{h \rightarrow 0}{\longrightarrow} & 0, \\
\left\|\left\{I_{U}-\bar{P}_{h} \bar{R}_{h}\right\} \psi\right\|_{U} \underset{h \rightarrow 0}{\longrightarrow} & 0 .
\end{array}
$$

REMARK 1. Note that the convergence hypotheses provided in Eq. (2.4) (Eq. (2.5) respectively) is not uniform with respect to $\phi \in X \quad(\psi \in U$ respectively).

For every $h \in\left(0, h_{0}\right)$, the vector spaces $X_{h}$ and $U_{h}$ are endowed with the norm $\|\cdot\|_{X_{h}}$ and $\|\cdot\|_{U_{h}}$ defined as follows

$$
\left\|\phi_{h}\right\|_{X_{h}} \triangleq\left\|P_{h} \phi_{h}\right\|_{X},\left\|\psi_{h}\right\|_{U_{h}} \triangleq\left\|\bar{P}_{h} \psi_{h}\right\|_{U} .
$$

Remark 2. With the endowed norms defined in (2.6), it is obvious that $P_{h}$ and $\bar{P}_{h}$ are both linear bounded operators satisfying

$$
\left\|P_{h}\right\|_{\mathcal{L}\left(X_{h}, X\right)}=\left\|\bar{P}_{h}\right\|_{\mathcal{L}\left(U_{h}, U\right)}=1 .
$$


Remark 3. By using the Banach-Steinhaus Theorem, Condition 2 in Assumption 1 implies that both $R_{h}$ and $\bar{R}_{h}$ are linear bounded operators, whose bounds are uniform in $h$. That is, for all $h \in\left(0, h_{0}\right)$, there exists $b_{R}>0$, independent of $h$, such that

$$
\left\|R_{h}\right\|_{\mathcal{L}\left(X, X_{h}\right)} \leq b_{R}, \quad\left\|\bar{R}_{h}\right\|_{\mathcal{L}\left(X_{h}, X\right)} \leq b_{R}
$$

For every $h \in\left(0, h_{0}\right)$, we define the approximation operator $A_{h}^{*}: X_{h} \rightarrow X_{h}$ of $A^{*}$ and $B_{h}^{*}: X_{h} \rightarrow U_{h}$ of $B^{*}$, by $A_{h}^{*}=R_{h} A^{*} P_{h}$ and $B_{h}^{*}=R_{h} B^{*} \bar{P}_{h}$. We set $A_{h}=\left(A_{h}^{*}\right)^{*}$ and $B_{h}=\left(B_{h}^{*}\right)^{*}$ with respect to the pivot space $X$ and $U$.

Together with a "good" space discretization, a "good" time discretization is also needed.

Assumption 2. [Time approximation] Let $A_{h}$ and $B_{h}$ defined in Assumption 1. For any $h \in\left(0, h_{0}\right)$, under an appropriate Courant-Friedrichs-Lewy (CFL) condition, there exist $\Delta t_{0}^{*}(h)>0$ and $\rho_{h}(\cdot) \in \mathcal{K}_{\infty}$ such that for all $\Delta t \in\left(0, \Delta t_{0}^{*}(h)\right)$, for any $\varphi_{h} \in X_{h}$ and $\nu_{h} \in U_{h}$, the following conditions hold

$$
\begin{gathered}
\left\|\left\{e^{A_{h} \Delta t}-A_{h, \Delta t}^{a}\right\} \varphi_{h}\right\|_{X_{h}} \leq \Delta t \rho_{h}(\Delta t)\left\|\varphi_{h}\right\|_{X_{h}}, \\
\left\|\left\{B_{h} \Delta t-B_{h, \Delta t}^{a}\right\} \nu_{h}\right\|_{X_{h}} \leq \Delta t \rho_{h}(\Delta t)\left\|\nu_{h}\right\|_{U_{h}} .
\end{gathered}
$$

Moreover, for any $t>0$, there exists a positive constant $\bar{B}_{A, a}=\bar{B}_{A, a}(t, h)$ such that

$$
\left\|\left(A_{h, \Delta t}\right)^{j}\right\|_{X_{h}} \leq \bar{B}_{A, a}, \forall j \in \mathbb{N}, \Delta t \in[0, t], \Delta t \in\left(0, \Delta t_{0}^{*}(h)\right) .
$$

When $A_{h}$ is obtained after space semi-discretization (for instance by a finite difference method), then $A_{h, \Delta t}^{a}$ and $B_{h, \Delta t}^{a}$ become

$$
A_{h, \Delta t}^{a}=\Delta t A_{h}+I_{X_{h}}, B_{h, \Delta t}=\Delta t B_{h}
$$

which satisfy Assumption 2 with $\rho_{h}(\Delta t)=\max \left\{\left\|A_{h}\right\|_{X_{h}}^{2}, 1\right\} \Delta t$.

REMARK 4. Time discretization is performed after the space discretization. In general, the CFL condition (see discussions on CFL conditions in [10, 15]) is required in order to guarantee that numerical approximations after space and time discretizations are "uniformly bounded" on compact intervals (or stable). By using the well-known Lax-Richtmyer Equivalence Theorem (see, for example [25]), the CFL condition can ensure that solutions of numerical approximations well approximate solutions of the exact continuous-time infinite-dimensional system. The CFL condition requires that the time discretization parameter $\Delta t$ to be sufficiently smaller than the space discretization parameter $h$. Therefore, in numerical discretization schemes, $h$ is chosen first and then $\Delta t$ is chosen accordingly.

Notice that both Assumptions 1 and 2 are rather general. For instance, Assumption 1 holds for almost all of the classical numerical space semi-discretization approximation schemes such as finite-difference methods, finite-element methods, Galerkin methods, spectral methods and so on.

2.2. Controller design. Once a family of finite-dimensional discrete-time numerical approximation systems (1.4) are obtained, the control input $u_{h, \Delta t}$ is designed to stabilize the approximation system (1.4). A family of "feedback" controllers are 
used in this paper, i.e., $u_{h, \Delta t}(j \Delta t)=K_{h, \Delta t} x_{h}^{a}(j \Delta t)$. The closed-loop of the approximation system (1.4) becomes

$$
\begin{aligned}
x_{h}^{a}((j+1) \Delta t) & =A_{h, \Delta t}^{a} x_{h}^{a}(j \Delta t)+B_{h, \Delta t}^{a} u_{h, \Delta t}(j \Delta t) \\
& =\left(A_{h, \Delta t}^{a}+B_{h, \Delta t}^{a} K_{h, \Delta t}\right) x_{h}^{a}(j \Delta t), x_{h}^{a}(0) \in X_{h}, \forall j \in \mathbb{N} .
\end{aligned}
$$

The feedback gain operator $K_{h, \Delta t}$ maps from $X_{h}$ to $U_{h}$ and is parameterized by the discretization parameters $(h, \Delta t)$. As discussed in the introduction, it is assumed that we have "good" controllers that can achieve some nice "uniform properties" of approximate models.

Assumption 3. The family of finite-dimensional discrete-time linear approximate models (2.12) are exponentially stable, uniformly in small $h$. That is, let $M_{a}$ and $\lambda_{a}$ be positive constants, there exists $h_{1}^{*}>0$ such that, for any $h \in\left(0, h_{1}^{*}\right)$, there exists $\Delta t_{1}^{*}(h)>0$ such that for any $\Delta t \in\left(0, \Delta t_{1}^{*}(h)\right)$, the solutions of systems (2.12), denoted as $x_{h}^{a}(j \Delta t)=x_{h}^{a}\left(j \Delta t ; x_{h}^{a}(0)\right)$, satisfy

$$
\left\|x_{h}^{a}\left(j \Delta t ; x_{h}^{a}(0)\right)\right\|_{X_{h}} \leq M_{a} e^{-\lambda_{a} j \Delta t}\left\|x_{h}^{a}(0)\right\|_{X_{h}}, \quad \forall j \in \mathbb{N} .
$$

REMARK 5. The stability property in Assumption 3 is only uniform in small $h$, though the choices of $M_{a}$ and $\lambda_{a}$ do not depend on either $\Delta t$ or $h$. As indicated in the introduction, Assumption 3 is a basic assumption in the proposed controller design method. We do not discuss, in the present paper, how to derive Assumption 3 in this work.

In [12, 33, 43, 44], numerical viscosity was employed to ensure uniform stability of a family of finite-dimensional approximations. Adding such a viscosity in numerical schemes can ensure that Assumption 3 holds.

For simplicity, $x_{h}^{a}\left(j \Delta t ; x_{h}(0)\right)$, the trajectories of the closed-loop system (2.12) can be represented as $\tilde{S}_{h, \Delta t}(j \Delta t) x_{h}(0)$ where $\tilde{S}_{h, \Delta t}$ is the semigroup generated by (2.12). By using Assumption 3, it can be derived that

$$
\left\|\tilde{S}_{h, \Delta t}(j \Delta t)\right\|_{X_{h}} \leq M_{a} e^{-\lambda_{a} j \Delta t}
$$

Assumption 3 is consistent with numerical discretization (Assumption 1 and Assumption 2) as the choice of $\Delta t_{1}^{*}$ depends on the choice of $h$.

Notice that the time discretization parameter $\Delta t$ has to be "different" from the sampling period $T$. The CFL condition requires that the choice of a proper $\Delta t$ depends on the choice of $h$ in order to ensure that trajectories of approximate models can well approximate trajectories of the exact model. Usually, $\Delta t$ is much smaller than a small space discretization parameter $h$. Since the sampling period cannot be arbitrarily small due to hardware limitation, the time discretization parameter and the sampling period must be different. The sampling period $T$ is typically much larger than $\Delta t$. To simplify the presentation, we assume that the ratio between $T$ and $\Delta t$ is an integer, i.e. $\frac{T}{\Delta t}=N, N \in \mathbb{N}, N \geq 1^{2}$.

After mapping from $U_{h}$ to $U$ by using the operator $\bar{P}_{h}$ (see, Assumption 1) and with the consideration of the sampling period $T$, we have

$$
u_{T}=u_{T, h, \Delta t}\left(k ; x_{0}\right)=\bar{P}_{h} K_{h, \Delta t} x_{h}^{a}\left(j N \Delta t ; x_{h}^{a}(0)\right),
$$

\footnotetext{
${ }^{2}$ The result can be extended when $T \in[N \Delta t,(N+1) \Delta t)$ with a slight modification of the proof.
} 
where $x_{h}^{a}\left(j \Delta t ; x_{h}^{a}(0)\right) \in X_{h}$ is the solution obtained from (2.12), satisfying Assumption 3 . With the control sequences (2.15), the sampled-data system (1.3) thus becomes

$$
\dot{x}(t)=A x(t)+B \bar{P}_{h} K_{h, \Delta t} u_{T, h, \Delta t}\left(k ; x_{0}\right) .
$$

The controller $u_{T, h, \Delta t}\left(k ; x_{0}\right)$ in $(2.15)$ is generated from a family of finite-dimensional discrete-time approximate models easy to implement in practice.

Control objective. Assume Assumptions 1-3 hold. This paper aims at providing sufficient conditions to ensure that trajectories of (2.16) converge to a neighborhood of the origin.

The sampled-data system (2.16) can be re-written as

$$
\dot{x}(t)=A x(t)+\bar{B} \bar{u}_{h}(k T),
$$

with $\bar{B} \triangleq B \bar{P}_{h}$ and $\bar{u}_{h} \triangleq K_{h, \Delta t} u_{T, h, \Delta t}\left(k ; x_{0}\right)$. Since the controller $\bar{u}_{h}$ belongs to a finite-dimensional space $X_{h}$, the control objective consists of finding sufficient conditions insuring that the controller $\bar{u}_{h}$ can drive exponentially an infinite-dimensional sampled-data system (1.3) to a neighborhood of the origin.

However, Assumptions 1-3 are not strong enough to ensure that the trajectories of the infinite-dimensional sampled-data system (2.16) will converge. On one hand, if there are infinitely many unstable modes in the system (2.17), it is not possible to design a finite-dimensional controller that can stabilize it, as pointed out in [36]. On the other hand, it is well-known that, for wave-like systems, numerical approximations may generate spurious solutions that do not exist in the exact model, thus leading to divergent trajectories, as discussed in $[12,43,44]$ and references therein. Other sufficient conditions are therefore needed.

Theorem 3.3 provides sufficient conditions to ensure that, for any well-behaved initial condition $x_{0}$, the control input $u_{T, h, \Delta t}\left(k ; x_{0}\right)$ can drive trajectories of the sampleddata system (2.16) to some neighborhood of the origin. The size of the neighborhood can be chosen arbitrarily small by tuning the parameters $(T, h, \Delta t)$ properly and choosing the appropriate filtering process with respect to this initial condition.

3. Sufficient Conditions and Main Results. This section discusses sufficient conditions that can ensure that the controller (2.15) can gradually move trajectories of the sampled-data system (2.16) to some neighborhood of the origin. It is followed by the statement of the main results (Theorems 3.3 and 3.4). Moreover, is also discussed the necessity of the conditions required in the statement of the theorems.

3.1. The existence of $\epsilon$-filtering. In this work, two slightly different $\epsilon$-filtering assumptions will be provided, the weaker one and the strong one. We start with the latter one.

Assumption 4. Let $\epsilon>0$ be arbitrary. Then, there exists $\mathcal{F}=\mathcal{F}(\epsilon)$, a linear continuous endomorphism of $X$, and $E=E(\epsilon)$, a finite-dimensional subspace of $X$, such that the following conditions hold:

(a) $X=E \bigoplus F$, where $F=F(\epsilon)$ is a closed subspace of $X$;

(b) there exists bounded linear operators $P_{E} \in \mathcal{L}(E, X)$ and $R_{E} \in \mathcal{L}(X, E), P_{F} \in$ $\mathcal{L}(F, X)$ and $R_{F} \in \mathcal{L}(X, F)$ such that $(i) R_{E} P_{E}=I_{E}$ and $R_{F} P_{F}=I_{F}$, where $I_{E}$ and $I_{F}$ are the identity operators on $E$ and $F$ respectively, (ii) for $x^{E} \in E$ and $x^{F} \in F,\left\|x^{E}\right\|_{E} \triangleq\left\|P_{E} x^{E}\right\|_{X}$ and $\left\|x^{F}\right\|_{F} \triangleq\left\|P_{F} x^{F}\right\|_{X}$ respectively. In that way, one has $\left\|P_{E}\right\|_{\mathcal{L}(E, X)}=\left\|P_{F}\right\|_{\mathcal{L}(F, X)}=1$;

(c) for every $x \in X,\left\|R_{F} \mathcal{F} x\right\|_{F}=\left\|P_{F} R_{F} \mathcal{F} x\right\|_{X} \leq \epsilon\|x\|_{X}$ 
(d) for every $x \in E$ ( $x \in F$ respectively), one has $A x \in E$ (Ax $\in F$ respectively), that is, $A$ induces two operators $A_{E}: E \rightarrow E$ and $A_{F}: F \rightarrow F$, generating $C_{0}$-semigroups $S_{E}(t)$ and $S_{F}(t)$ respectively;

(e) the semigroup $S_{F}(t)$ is a uniformly bounded linear operator, i.e., there exists $b_{F}>$ 0 such that $\left\|S_{F}(t)\right\|_{\mathcal{L}(X)} \leq b_{F}$.

We are now in a position to define precisely the filtering procedure used in this paper.

Definition 3.1. A element $x \in X$ is said filtered if $\mathcal{F}(\epsilon) x=x$, i.e, $x \in$ $\operatorname{Ker}\left(\mathcal{F}(\epsilon)-I_{X}\right)$, where $\mathcal{F}$ is defined in Assumption 4.

Note that Condition $(d)$ in Assumption 4 implies that the infinitesimal generator A can be decomposed as $A=\left[\begin{array}{cc}A_{E} & 0 \\ 0 & A_{F}\end{array}\right]$. In particular, System (1.1) can be re-written as follows (see [38, Page 711] for more details)

$$
\begin{aligned}
& \dot{x}^{E}=A_{E} x^{E}+B_{E} u(t), \quad B_{E}=R_{E} B, \\
& \dot{x}^{F}=A_{F} x^{F}+B_{F} u(t), \quad B_{F}=R_{F}\left(I_{X}-P_{E} R_{E}\right) B .
\end{aligned}
$$

Intuitively, when diagonalizing (if possible) the $C_{0}$-semigroup $S(t)$, the subspace $E$ contains a finite number of unstable modes and $F$ contains an infinite number of stable (non-positive) modes. Note that, such a decomposition method is widely used in the controller design of PDEs. For example, it was used in a pole shifting process in [38] and in structural assignment for parabolic equations using Dirichlet boundary feedback in [23] and sampled-data control of infinite-dimensional systems in [28]. It was also used in [7] for stabilization of semilinear heat equations and in [8] for stabilization of semilinear wave equations.

As discussed in [38], $A_{E}$ can be generated by spanning the first $M$ unstable eigenvalues of the operator $A$, where $M$ is some fixed integer, and $A_{F}$ can be generated by spanning infinitely many stable eigenvalues of $A$. If $A_{E}$ is known exactly, by constructing appropriate feedback control laws to stabilize $M$ unstable modes without moving the others, it is possible to obtain the practical stability properties of the system (2.16) when the initial condition is filtered. However $E$ may not be known a priori in most applications. For example, engineering practitioners sometimes can "guess" what $E$ should be with some uncertainty measure (noise on estimation), up to a certain precision level $\epsilon$, as indicated in Condition (b) in Assumption 4.

Assumption 4 also requires the existence of $\epsilon$-filtering for any given $\epsilon$ (the quality of the filter). That is, for any $\epsilon>0$ arbitrary, it is possible to construct a welldesigned filter so that Condition (b) holds. This is a relatively strong assumption leading to a strong result in which $\Delta$ and $\nu$ can be any positive constants, as shown in Theorem 3.3: how to choose $\epsilon$ for the filter depends on the set in which the initial condition stays and the neighborhood of the origin to which the solutions of System (2.2) converge. The smaller the $\nu$ (the size of the neighborhood) and the larger the $\Delta$ (the set containing the initial condition), the smaller the $\epsilon$ is needed, requiring a better filtering process (see Theorem 1).

In practice, it is not always possible to obtain a filtering process achieving the given precision requirement $\epsilon$. A weaker version of Assumption 4 can be used when practitioners know roughly on the space $E$ and can guess the size of $\epsilon$ with respect to the $E$.

Assumption 5. There exist a linear continuous operator $\mathcal{F}: X \rightarrow X$, a subspace $E=E \subset X$ with $\operatorname{dim}(E)<\infty$ and a positive constant $\epsilon=\epsilon(\mathcal{F})$, such that conditions (a)-(e) in Assumption 4 hold. 
With a weaker filtering assumption, a weaker result for System (2.16) will be obtained, as shown in Theorem 3.4. It states that $\nu$, the size of the neighborhood of the origin (or offset) will be determined by $\epsilon$. The better is the filter (the smaller $\epsilon$ ), the smaller $\nu$ will be obtained in practical stabilization. Designing a proper filtering process is natural in the control of the PDEs and the control performance depends on the choice of filtering processes. How to design this $\epsilon$-filtering is outside of the scope of this paper.

3.2. High frequency filtering property. If $A_{E}$ is known, System (1.1) can be practically stabilized by a finite-dimensional controller on $E$. However, $A_{E}$ is not completely known in general. The approximation of $E$ is known according to Assumption 4 with an approximation error majorized by $\epsilon$. Since $B$ is an unbounded control operator, the existence of $F$ can perturb stable modes of the sampled-data system (1.3) through $B$ and may lead to divergent trajectories. Therefore, the following assumption is also needed.

Assumption 6. "High frequency filtering property" (HFFP) with respect to $\epsilon$ filtering is satisfied for System (1.1). That is, let $\epsilon$ defined in Assumption 4 or Assumption 5 , for any $y(\cdot) \in \mathcal{L}^{2}([0, t], U)$ satisfying $\|y(t)\|_{U} \leq e^{-\lambda_{y} t}, \forall t \geq 0$ for some positive $\lambda_{y}$, then the following inequality holds

$$
\left\|\int_{0}^{t} S_{F}(t-s) R_{F}\left(I_{X}-P_{E} R_{E}\right) B y(s) d s\right\|_{X} \leq \epsilon, \forall t \geq 0 .
$$

Assumption "HFFP" is new and plays a crucial role in our general problem setting. It reflects what has been done by E. Zuazua and his coauthors in their work in which filtering out high frequencies components is needed (see discussion in $[12,43,44,45])$. It is also consistent with what engineers are always doing in practice, when applying filters to their process to regularize their data.

Notice that Assumption "HFFP" is not restrictive in the control of PDEs. For instance, if $A$ is analytic and $B$ is admissible (e.g., parabolic PDEs), then Assumption "HFFP" is always satisfied, as possible high frequency components are automatically damped out (see more discussion in [12]); if $A$ is not analytic (e.g. hyperbolic PDEs), a "uniform gap assumption" (see [33] where it is combined with a moment method and also $[8,38]$ ) can ensure that Assumption "HFFP" holds.

3.3. Uniform boundedness of $K_{h, \Delta t}$. The following assumption is always needed when the direct method is employed.

Assumption 7. There exists $h_{0}>0$ such that for any $h \in\left(0, h_{0}\right)$, there exist $\Delta t_{K}^{*}(h)>0$ such that for all $\Delta t \in\left(0, \Delta t_{K}^{*}(h)\right)$, there exists $B_{K}>0$ such that the family of feedback control operators satisfy $\left\|K_{h, \Delta t}\right\|_{\mathcal{L}\left(X_{h}, U_{h}\right)} \leq B_{K}$.

REMARK 6. Assumptions 3 and 7 are satisfied with the Riccati procedure that appears in the LQR optimal control problems (See discussion and results in [3, 1, 13, 17, 24, 22, 33]).

3.4. Uniform Hurwitzian property. Assumptions 1 and 2 ensure the existence of "good" numerical algorithms. Assumptions 3 and 7 guarantee the existence of good finite-dimensional controllers $u_{h, \Delta t}$. Assumptions 4 (or 5 ) and 6 ensure that "good" filtering process is available. However, it is still not enough to ensure that trajectories of the sampled-data system (2.16) do converge. The compatibility requirement (or uniform Hurwitzian property) is also needed. The space semi-discretization 
models are used in this assumption:

$$
\dot{x}_{h}(t)=A_{h} x_{h}(t)+B_{h} K_{h, \Delta t} x_{h}(t),
$$

with the initial condition $x_{h}(0) \in X_{h}$. The solutions of (3.4) with the control input $u_{h}(t)$ are represented as $x_{h}\left(t ; x_{0}, u_{h}(t)\right)$.

Assumption 8. "Uniform Hurwitzian property" (UHP) is satisfied for System (2.16). That is, let $M_{E}$ and $\lambda_{E}$ be positive constants with $M_{E}>1$. Let the feedback control operators $K_{h, \Delta t}$ be defined in (2.12). Then, there exist $T_{E}^{*}>0$ and $h_{E}^{*}>0$ such that, for $T \in\left(0, T_{E}^{*}\right)$ and $h \in\left(0, h_{E}^{*}\right)$, there exists $\Delta t_{E}^{*}(h)$ such that, for $\Delta t \in$ $\left(0, \Delta t_{E}^{*}(h)\right)$ and $z_{0} \in E$, solutions of the following system, denoted as $z\left(t ; z_{0}\right)$,

$$
\dot{z}(t)=R_{E} P_{h} A_{h} R_{h} P_{E} z(t)+R_{E} P_{h} B_{h} K_{h, \Delta t} R_{h} P_{E} z(k T),
$$

with $z(0)=z_{0} \in E$, satisfy

$$
\left\|z\left(t ; z_{0}\right)\right\|_{E}=\left\|P_{E} S_{T, h}^{E}(t) z_{0}\right\|_{X} \leq M_{E} e^{-\lambda_{E} t}\left\|z_{0}\right\|_{E},
$$

where $S_{T, h}^{E}$ represents a family of semigroups generated by System (3.5).

Although Assumption 8 plays an important role in our results, it is not always easy to verify whether this assumption holds or not in practice. The following proposition provides a sufficient condition which is easier to check to ensure that Assumption 8 holds.

Proposition 3.2. If the family of matrices $R_{E} P_{h}\left(A_{h}+B_{h} K_{h, \Delta t}\right) R_{h} P_{E}$ is uniformly Hurwitz for $h$ small enough and $\Delta t$ selected according to Assumptions 1 and 2, then Assumption 8 holds true.

Sketch of Proof: Since the matrix $R_{E} P_{h}\left(A_{h}+B_{h} K_{h, \Delta t}\right) R_{h} P_{E}$ is uniformly Hurwitz, the stability property is independent of the choice of $h$ and $\Delta t$. Applying a standard emulation result [31], or indirect method as in [5] leads to the conclusion.

Remark 7. The filtered feedback matrix $R_{E} P_{h}\left(A_{h}+B_{h} K_{h, \Delta t}\right) R_{h} P_{E}$ can be treated as the matrix $A_{h}+B_{h} K_{h, \Delta t}$ viewed "through E". Proposition 3.2 is a general emulation result on the sampled-data control of finite-dimensional systems with sampling period T. Note that, since the stability properties of $R_{E} P_{h}\left(A_{h}+B_{h} K_{h, \Delta t}\right) R_{h} P_{E}$ are uniform in $h$, the choice of $T^{*}$ is independent of the choice $h$.

REMARK 8. Assumption 8 is satisfied if the discretization scheme is built on $E$ and such numerical schemes are called spectral, or modal, that is, $E \subset \operatorname{Im}\left(R_{h}\right)$, for $h$ small enough, cf. [7, 8].

Although the controller (2.16) is not a feedback, Assumption 8 ensures that all unstable modes can be exponentially stabilized by controllers designed for numerical approximations.

3.5. Main result. The main result of this paper is stated as follows.

Theorem 3.3. Let $(\Delta, \nu)$ be positive constants. Assume that Assumptions 1, 2, 3, 4, 6, 7 and 8 hold true with $\epsilon$ defined in Assumptions 4-6. Then, there exists $T^{*}>0$, $h^{*}>0$ such that, for $T \in\left(0, T^{*}\right)$ and $h \in\left(0, h^{*}\right)$, there exists $\Delta t^{*}(h)>0$ and two positive constants $M$ and $\lambda$ such that, for $\Delta t \in\left(0, \Delta t^{*}(h)\right), x_{0} \in B_{\Delta} \cap \operatorname{Ker}\left(\mathcal{F}(\epsilon)-I_{X}\right)$, the trajectories of (2.16), denoted as $x(t)=x\left(t ; x_{0}, u_{T, h, \Delta t}\left(k ; x_{0}\right)\right)$, are well-defined on $\mathbb{R}^{+}$and satisfy

$$
\left\|x\left(t ; x_{0}, u_{T, h, \Delta t}\left(k ; x_{0}\right)\right)\right\|_{X} \leq M e^{-\lambda t}\left\|x_{0}\right\|_{X}+\nu,
$$

for all $t \in[k T,(k+1) T), k \in \mathbb{N}$. 
The above stability result belongs to the realm of "practical exponential stability". In general, obtaining "practical stability" is easier than "stability" (see discussion in [44]). In the context of the paper, it is not always possible to obtain exponentially stability properties when the direct method is applied to general sampled-data infinite-dimensional systems. Due to the existence of the sampling mechanism, numerical approximation errors as well as the estimation error $\epsilon$ on $E$, practical stability properties are (probably) the best result that one could obtain.

The result proved in Theorem 3.3 extends previous results obtained in [28] in two ways: $(i)$ in the aforementioned paper, only sampling is considered and the controller is designed for continuous-time systems; $(i i)$ in the present paper, the system considered is more general ( $\mathrm{A}$ is not analytic and $B$ is unbounded) than that in [28].

Normally, one cannot get the suitable filtering process to achieve the required precision $\epsilon$, which is determined by $\nu$ and $\Delta$ (see Theorem 3.3). In most of situations, engineers have a filter with known " $\epsilon$ ", and then the size of the neighborhood depends on the quality of the filter (see Assumption 5). When a weaker assumption is used, it leads to a weaker result given next.

TheOrem 3.4. Let $(\Delta, \epsilon)$ be positive constants. Assume Assumptions 1, 2, 3, 5, 6,7 and 8 hold true. Then, there exists $T^{*}>0, h^{*}>0$ such that, for $T \in\left(0, T^{*}\right)$, and $h \in\left(0, h^{*}\right)$, there exists $\Delta t^{*}(h)>0$, two positive constants $M$ and $\lambda$ and a class $\mathcal{K}_{\infty}$-function $\gamma$ such that, for $\Delta t \in\left(0, \Delta t^{*}(h)\right)$ and $x_{0} \in B_{\Delta} \cap \operatorname{Ker}\left(\mathcal{F}(\epsilon)-I_{X}\right)$, the trajectories of (2.16), denoted as $x(t)=x\left(t ; x_{0}, u_{T, h, \Delta t}\left(k ; x_{0}\right)\right)$ are well-defined on $\mathbb{R}^{+}$ and satisfy

$$
\left\|x\left(t ; x_{0}, u_{T, h, \Delta t}\left(k ; x_{0}\right)\right)\right\|_{X} \leq M e^{-\lambda t}\left\|x_{0}\right\|_{X}+\gamma(\epsilon)
$$

for all $t \in[k T,(k+1) T), k \in \mathbb{N}$.

Sketch of proof. The proof of Theorem 3.4 is similar to the proof of Theorem 3.3, with a slight modification of Step 1.

REMARK 9. In [22], Labbé and Trélat discussed necessary and sufficient conditions under which uniform controllability properties of a family space semi-discretized approximations imply the controllability properties of the exact model (1.1) for parabolic systems. The main result in their paper [22, Theorem 3.1] implies Theorem 3.3 in the parabolic case, though the complete argument of this implication would require several developments.

REMARK 10. When the infinitesimal generator $A$ in (1.1) is analytic, i.e. System (1.1) is parabolic, HFFP condition is automatically satisfied. Assumption 3 and Assumption 7 are satisfied with the Riccati procedure (see discussion in [3, 1, 13, 17, 24, 22] and references therein). Under such a situation, the filtering process is not required since high frequency components are naturally damped. Sharp estimates of convergence of $S_{h}(t)$ to $S(t)$ therefore exist (see [24, Chapter 4]), which can greatly simplify the proof of our main result.

REMARK 11. If suitable numerical viscosity terms are added in the numerical schemes in our main result, Assumptions 3 and 7, 8 and 6 hold (see discussion in [33] for more details). Therefore, by choosing appropriate numerical schemes, the assumptions in Theorem 3.3 or Theorem 3.4 are not restrictive.

Theorem 3.3 provides a useful guideline for engineers to choose appropriate numerical schemes ( $h$ and $\Delta t$ ), sampling period $T$ and filtering processes. There are four design parameters $(T, h, \Delta t, \epsilon)$ that are determined by the performance requirement $(\Delta, \nu)$. Therefore, how to design these parameters is of great importance to ensure that the proposed method can work for a general sampled-data LDPS. 
3.6. Discussion on necessity of the assumptions. Although the proposed method (designing controllers for approximate models) is widely used in engineering applications, assumptions in Theorem 3.3 may be very hard to check in practice. However, our result shed the light on how to properly design sampled-data controllers for infinite-dimensional systems by using numerical approximate models. Furthermore, Assumptions 4-8 are not only sufficient, but also necessary. If one of them fails, then counterexamples can be found in literature, as explained below.

Assumption 4 or Assumption 5. The initial condition $x_{0}$ must be filtered. The role of $\epsilon$-filtering is to filter out the high frequency components in space for the given initial condition $x_{0}$ of the exact system (1.1). It is important to note that the assumption of $\epsilon$-filtering (Assumption 4 or Assumption 5) and filtering out high frequency components of initial conditions is necessary. Counterexamples can be found when wave-like equations are considered. It is well-known that, for hyperbolic systems, high frequency components of initial conditions may not be damped and will interfere with the numerical discretization, generating spurious oscillation and leading to divergent trajectories. Such discussion can be found in [44, 43, 12].

Assumption 6. That assumption cannot be removed in general, as $A$ is not restricted to be analytic and $B$ is an unbounded operator. For example, when the hyperbolic PDEs are considered, "resonance" phenomena may occur if Assumption "HFFP" is not satisfied. Unstable trajectories thus could be obtained (see discussion in [33] and references therein).

Assumption 7. If that assumption is not satisfied, when $h$ and $\Delta t$ tend to zero, $\left\|K_{h, \Delta t}\right\|_{X_{h}}$ may tend to infinity, leading to divergent control input $u_{T, h, \Delta t}\left(k ; x_{0}\right)$ (see discussion in [44]). Uniform boundedness of $K_{h, \Delta t}$ is thus necessary.

Assumption 8. That assumption actually plays a crucial role in Theorem 3.3. Intuitively, if the numerical schemes are not compatible, i.e., some unstable modes cannot be detected and stabilized by numerical approximations, it is not possible to obtain convergent trajectories of the sampled-data system (1.3). If the discretization scheme is not spectral (i.e., Assumption 8 does not hold), then the trajectories of the sampled-data system (1.3) may diverge, see, for example, [41] in which finite differences are used.

\section{Proof of the main result.}

4.1. Propositions and Corollaries needed in the proof of Theorem 3.3. Introducing two different control inputs for the space discretization system (3.4):

$$
\begin{aligned}
& u_{1}(t) \triangleq K_{h, \Delta t} x_{h}(t), \\
& u_{2}(t) \triangleq K_{k, \Delta t} x_{h}(k T), \quad \forall t \in[k T,(k+1) T), \forall k \in \mathbb{N},
\end{aligned}
$$

then $x_{h}\left(t ; x_{h}(0) ; u_{1}(t)\right)$ and $x_{h}\left(t ; x_{h}(0), u_{2}(t)\right)$ represent solutions of (3.4) with two different control inputs respectively. The following propositions are needed in the proof of the main result.

Proposition 4.1. Consider the following linear finite-dimensional continuoustime system

$$
\dot{x}(t)=\Phi x(t), \quad x(0) \in \mathbb{R}^{n},
$$

and its time-discretization

$$
x(k+1)=\Phi_{T}^{a} x(k), \quad x(0) \in \mathbb{R}^{n} .
$$


Then, we use $\phi^{e}(t ; x(0))$ and $\phi_{T}^{a}(k ; x(0))$ to denote the solutions of (4.3) and (4.4) respectively. Let $\delta>0$ be arbitrary. Assume that the following conditions hold.

1. There exist $T_{0}>0$ and $\rho(\cdot) \in \mathcal{K}_{\infty}$ such that for all $T \in\left(0, T_{0}\right)$

$$
\left\|e^{\Phi T}-\Phi_{T}^{a}\right\|_{\mathbb{R}^{n}} \leq T \rho(T) .
$$

2. For any $t>0$, there exists $B_{A, a}(t)>0$ such that

$$
\left\|\left(\Phi_{T}^{a}\right)^{k}\right\|_{\mathbb{R}^{n}} \leq B_{A, a}(t), \forall k \in \mathbb{N} \text { such that } k T \in[0, t] .
$$

Then, there exists $T^{*}>0$ such that, for $T \in\left(0, T^{*}\right)$, the following holds

$$
\left\|\phi^{e}(k T ; x(0))-\phi_{T}^{a}(k ; x(0))\right\|_{\mathbb{R}^{n}} \leq \delta\|x(0)\|_{\mathbb{R}^{n}}, \quad \forall k \in \mathbb{N}, k T \in[0, t] .
$$

Proof of Proposition 4.1: Consider $\delta>0$ and $t>0$. Define

$$
T^{*} \triangleq \min \left\{\rho^{-1}\left(\frac{\delta}{B_{A, a}(t) \cdot e^{\Phi t} \cdot t}\right), T_{0}\right\},
$$

where $\rho(\cdot)$ and $T_{0}$ are defined in Condition 1 above.

For any $k T \in[0, t], k \in \mathbb{N}$, it follows that

$$
\begin{aligned}
& \left\|\phi^{e}(k T ; x(0))-\phi_{T}^{a}(k ; x(0))\right\|_{\mathbb{R}^{n}}=\left\|\sum_{i=0}^{k-1}\left(\Phi_{T}^{a}\right)^{i}\left(\Phi_{T}^{a}-e^{\Phi T}\right) e^{\Phi((k-1-i) T)} x(0)\right\|_{\mathbb{R}^{n}} \\
\leq & B_{A, a}(t) \sum_{i=0}^{k-1}\left\|\left(\Phi_{T}^{a}-e^{\Phi T}\right)\right\|_{\mathbb{R}^{n}} e^{\Phi t}\|x(0)\|_{\mathbb{R}^{n}} \\
\leq & B_{A, a}(t) \cdot e^{\Phi t} \cdot(k-1) \cdot T \cdot \rho(T) \cdot\|x(0)\|_{\mathbb{R}^{n}} \\
\leq & B_{A, a}(t) \cdot e^{\Phi t} \cdot t \cdot \rho(T) \cdot\|x(0)\|_{\mathbb{R}^{n}}=\delta\|x(0)\|_{\mathbb{R}^{n}}, \forall T \in\left(0, T^{*}\right),
\end{aligned}
$$

which completes the proof of Proposition 4.1.

In order to extend Proposition 4.1 to an infinite-dimensional setting, a more general notation is used.

Definition 4.2. Let $\Theta$ be a compact subset of $\mathbb{R}^{n_{\theta}}$ where $n_{\theta} \in \mathbb{N}$. A parameterized set $\mathcal{S}_{1}$ of functions $x(\cdot): I \rightarrow X$ where $I$ is any subinterval of the form $\left[0, t_{*}\right)$, with $t_{*} \in[0, \infty]$, is said to be a flow if, for all $x_{0} \in X$ and $\theta \in \Theta$, the following holds.

1. For all $x_{0} \in X$ and $\theta \in \Theta$, the set $\mathcal{S}_{1}\left(x_{0}, \theta\right)$ is used to denote the functions $x(\cdot)$ verifying $x(0)=x_{0}$.

2. If $x(\cdot) \in \mathcal{S}_{1}\left(x_{0}, \theta\right)$ and $t_{1} \in \operatorname{domain}(x(\cdot))$, then $x\left(t_{1}+\cdot\right)$ belongs to $\mathcal{S}_{1}\left(x\left(t_{1}\right), \theta\right)$ for every $\theta \in \Theta$.

An auxiliary flow $\mathcal{S}_{2}$ will be considered with no dependence with respect to the parameter $\theta$, wherwhich starts from the same initial condition $x_{0}$ of $\mathcal{S}_{1}$, is denoted as $\mathcal{S}_{2}\left(x_{0}\right)$. With the above notations, Proposition 4.1 will be extended as follows.

Proposition 4.3. Assume that the following conditions hold:

1. there exists three positive real numbers $M_{1}, \lambda_{1}$ and $\theta_{a}>0$ such that, for $x_{0} \in$ $X, \theta \in \Theta$ satisfying $\|\theta\|_{\mathbb{R}^{n_{\theta}}} \in\left(0, \theta_{a}\right)$ and $x(\cdot) S_{1}\left(x_{0}, \theta\right)$, one has $\|x(t)\|_{X} \leq$ $M_{1} e^{-\lambda_{1} t}\left\|x_{0}\right\|_{X}$ for all $t \in I$

2. the solutions of the flows $\mathcal{S}_{1}\left(x_{0}, \theta\right)$ and $\mathcal{S}_{2}\left(x_{0}\right)$ can be made arbitrarily close on compact time intervals. That is, given any $\delta>0$ and $t \in I$, there exists $\theta_{b}>0$ such that, for $\theta \in \Theta$ satisfying $\|\theta\|_{\mathbb{R}^{n_{\theta}}} \in\left(0, \theta_{b}\right), x(\cdot) \in \mathcal{S}_{1}\left(x_{0}, \theta\right)$ and $y(\cdot) \in \mathcal{S}_{2}\left(x_{0}\right)$, the following holds

$$
\|x(s)-y(s)\|_{X} \leq \delta\left\|x_{0}\right\|_{X}, \forall s \in[0, t] ;
$$


3. the solutions of the flow $\mathcal{S}_{2}\left(x_{0}\right)$ are bounded on compact time intervals, that is, for any $t \in I$, there exists $L>0$ such that $\|y(s)\|_{X} \leq L\left\|x_{0}\right\|_{X}$, for all $s \in[0, t]$.

Then $\mathcal{S}_{2}\left(x_{0}\right)$ is exponentially stable.

Proof of Proposition 4.3: Let $c \in(0,1)$ and $\delta \in(0, c)$ be arbitrary. Let $M_{1}$ and $\lambda_{1}$ come from Item 1 of the proposition. Define $t \triangleq \frac{1}{\lambda_{1}} \ln \left(\frac{M_{1}}{c-\delta}\right)$ and let this $t$ and the given $\delta$ determine $\theta_{b}>0$ via Item (2) of the proposition. Consider $x_{0} \in X, \theta \in \Theta$ such that $\|\theta\|_{\mathbb{R}^{n_{\theta}}} \leq \theta^{*}=\min \left\{\theta_{a}, \theta_{b}\right\}$ and $x(\cdot) \in S_{1}\left(x_{0}, \theta\right)$. For simplicity, we assume that $I=\mathbb{R}_{+}$. Introduce now a sequence of times $t_{i} \triangleq i$, where $i=0,1,2, \ldots$ and consider the solution $y\left(t_{i+1}, x_{0}\right)$. Thanks to the second property of the flow, we can write that $y\left(t_{i+1}\right) \triangleq y\left(t_{i+1}, x_{0}\right)=y\left(t, y\left(t_{i}\right)\right)$. At each $t_{i}$, we reinitialize the flow $\mathcal{S}_{1}$ with $y\left(t_{i}\right)$, we denote it $x\left(t, y\left(t_{i}\right), \theta\right)$.

Then, we can write for all $t_{i}, x\left(t_{i}\right) \in X$ and $y(s) \in \mathcal{S}_{2}\left(y\left(t_{i}\right)\right)$ :

$$
\begin{aligned}
& \left\|y\left(t_{i+1}\right)\right\|_{X}=\left\|y\left(t, y\left(t_{i}\right)\right)\right\|_{X} \\
\leq & \left\|x\left(t, y\left(t_{i}\right), \theta\right)\right\|_{X}+\left\|x\left(t, y\left(t_{i}\right), \theta\right)-y\left(t, y\left(t_{i}\right)\right)\right\|_{X} \\
\leq & M_{1} \exp \left(-\lambda_{1} t\right)\left\|y\left(t_{i}\right)\right\|_{X}+\delta\left\|y\left(t_{i}\right)\right\|_{X} \\
= & (c-\delta)\left\|y\left(t_{i}\right)\right\|_{X}=c\left\|y\left(t_{i}\right)\right\|_{X} .
\end{aligned}
$$

Moreover, we can write that

$$
\left\|y\left(t_{i}\right)\right\|_{X} \leq c^{i}\left\|x_{0}\right\|_{X} \leq e^{-\lambda t_{i}}\left\|x_{0}\right\|_{X},
$$

where $\lambda \triangleq \ln (1 / c)$. Finally, using Item 3 of the proposition, we can write that, for $s \in\left[t_{i}, t_{i+1}\right]$,

$$
\begin{aligned}
& \|y(s)\|_{X} \leq L\left\|y\left(t_{i}\right)\right\|_{X} \leq L\left\|y\left(t_{i}\right)\right\|_{X} \leq L e^{-\lambda t_{i}}\left\|x_{0}\right\|_{X} \\
= & L e^{\lambda\left(s-t_{i}\right)} e^{-\lambda s}\left\|x_{0}\right\|_{X} \\
\leq & L \exp (\lambda t) e^{-\lambda s}\left\|x_{0}\right\|_{X} \leq M e^{-\lambda s}\left\|x_{0}\right\|_{X},
\end{aligned}
$$

which completes the proof of Proposition 4.3, with $M \triangleq L e^{\lambda t}$.

The following corollaries can be obtained from Propositions 4.1 and 4.3.

Corollary 4.4. Assume that Assumptions 1, 2 and 7 hold. Then there exist positive real numbers $M_{p, 1}, \lambda_{p, 1}, h_{p, 1}^{*}$ such that, for $h \in\left(0, h_{p, 1}^{*}\right)$, there exists $\Delta t_{p, 1}^{*}(h)>0$ so that, for $\Delta t \in\left(0, \Delta t_{p, 1}^{*}(h)\right)$, solutions of (3.4) with control inputs $u_{2}(\cdot)$ satisfy

$$
\left\|x_{h}\left(t, x_{h}(0), u_{2}(t)\right)\right\|_{X_{h}} \leq M_{p, 1} e^{-\lambda_{p, 1} t}\left\|x_{h}(0)\right\|_{X_{h}}, \quad \forall h \in\left(0, h_{p, 1}^{*}\right), t \geq 0 .
$$

Proof of Corollary 4.4: Let $h_{p, 1}^{*}=\min \left\{h_{1}^{*}, h_{0}\right\}$, where $h_{1}^{*}$ and $h_{0}$ are defined in Assumption 3 and Assumption 2 respectively. Let $t>0$ and $\delta>0$. Denote $\Psi_{h}^{e}=$ $A_{h}+B_{h} K_{h, \Delta t}$ and $\Psi_{h, \Delta t}^{a}=A_{h, \Delta t}^{a}+B_{h, \Delta t}^{a} K_{h, \Delta t}$. Then, one has

$$
\begin{aligned}
x_{h}^{a}(k+1) & =\left(A_{h, \Delta t}^{a}+B_{h, \Delta t}^{a} K_{h, \Delta t}\right) x_{h}^{a}(k)=\Psi_{h, \Delta t}^{a} x_{h}^{a}(k), \\
\dot{x}_{h}(t) & =\left(A_{h}+B_{h} K_{h, \Delta t}\right) x_{h}(t)=\Psi_{h}^{e} x_{h}(t), \quad u(t)=u_{1}(t)=K_{h, \Delta t} x_{h}(t) .
\end{aligned}
$$

The proof is completed by showing the following facts.

Fact 1 For any fixed $h \in\left(0, h_{p, 1}\right), A_{h} \in X_{h}$ and $B_{h} \in \mathcal{L}\left(U_{h}, X_{h}\right)$. 
Thus for any fixed $h$, there exists positive constants $B_{A, h}$ and $B_{B, h}$ such that

$$
\left\|A_{h}\right\|_{X_{h}} \leq B_{A, h}, \quad\left\|B_{h}\right\|_{\mathcal{L}\left(U_{h}, X_{h}\right)} \leq B_{B, h}
$$

Fact 2 From Assumption 7, it follows that $\left\|K_{h, \Delta t}\right\|_{\mathcal{L}\left(X_{h}, U_{h}\right)} \leq B_{K}$.

Fact 3 For any $h \in\left(0, h_{p, 1}^{*}\right)$, there exists $\bar{\rho}_{h}(\cdot) \in \mathcal{K}_{\infty}$ such that

$$
\left\|e^{\Psi_{h}^{e} \Delta t}-\Psi_{h, \Delta t}^{a}\right\|_{X_{h}} \leq \Delta t \bar{\rho}(\Delta t) .
$$

Proof of Fact 3: Let $\rho_{h}(\cdot)$ be defined in Assumption 2. Then, using Assumption 2, for any $h \in\left(0, h_{p, 1}^{*}\right)$, there exists a positive constant $\Delta t_{p, 1}^{*}(h)$ and class $\mathcal{K}_{\infty}$-functions $\bar{\rho}_{1, h}(\cdot)$ and $\bar{\rho}_{2, h}(\cdot)$ such that

$$
\begin{aligned}
& \left\|e^{A_{h} \Delta t} e^{B_{h} K_{h, \Delta t} \Delta t}-\Psi_{h, \Delta t}^{a}\right\|_{X_{h}} \\
\leq & \left\|e^{A_{h} \Delta t}\left(e^{B_{h} K_{h, \Delta t} \Delta t}-I_{X_{h}}-B_{h} K_{h, \Delta t} \Delta t\right)\right\|_{X_{h}}+\left\|e^{A_{h} \Delta t}-A_{h, \Delta t}^{a}\right\|_{X_{h}} \\
& +\left\|\left(e^{A_{h, \Delta t} \Delta t}-I_{X_{h}}\right) B_{h} K_{h, \Delta t} \Delta t\right\|_{X_{h}}+\left\|B_{h} K_{h, \Delta t} \Delta t-B_{h, \Delta t}^{a} K_{h, \Delta t}\right\|_{X_{h}} \\
\leq & \bar{\rho}_{1, h}(\Delta t) \Delta t+\rho_{h}(\Delta t) \Delta t+\bar{\rho}_{2, h}(\Delta t) \Delta t+\rho_{h}(\Delta t) \Delta t=\bar{\rho}(\Delta t) \Delta t .
\end{aligned}
$$

Fact 4 Using Assumption 2 , let $\delta>0$ and $t>0$. Then, for any $h \in\left(0, h_{p, 1}^{*}\right)$, there exists $\Delta t_{p, 1}^{*}(h)>0$ such that

$$
\left\|x_{h}^{a}(j)-x_{h}\left(j \Delta t ; x_{h}(0), u_{1}(t)\right)\right\|_{X_{h}} \leq \Delta\left\|x_{h}(0)\right\|_{X_{h}}, \forall j \in \mathbb{N}, \Delta t \in[0, t] .
$$

The proof of Corollary 4.4 can now be completed by applying Proposition 4.1 and Fact 4.

Corollary 4.5. Assume that Assumptions 1, 2 and 7 hold. Then, there exists positive constants $\left(M_{p, 2}, \lambda_{p, 2}\right), h_{p, 2}^{*}$ and $T_{p, 2}^{*}$ such that, for $h \in\left(0, h_{p, 2}^{*}\right)$ and $T \in$ $\left(0, T_{p, 2}^{*}\right)$, if one defines the piecewise-constant control $u_{2}$ by $K_{h, \Delta t} x_{h}(k T)$ on $[k T,(k+$ $1) T), k \in \mathbb{N}$, then the solutions of (3.4) corresponding to $u_{2}(\cdot)$ satisfy

$$
\left\|x_{h}\left(t ; x_{h}(0), u_{2}(k)\right)\right\|_{X_{h}} \leq M_{p, 2} e^{-\lambda_{p, 2} t}\left\|x_{h}(0)\right\|_{X_{h}}, \quad \forall t \geq 0 .
$$

Proof of Corollary 4.5: Let $h_{p, 2}^{*}=h_{p, 1}^{*}$, where $h_{p, 1}^{*}$ is defined in Corollary4.4. We first prove the following fact.

Fact a If $\delta>0$ and $t>0$, then there exists $T^{*}>0$ such that, for $T \in\left(0, T^{*}\right)$ and $h \in\left(0, h_{p, 2}^{*}\right)$, one has

$$
\left\|x_{h}\left(k T ; x_{h}(0), u_{1}(t)\right)-x_{h}\left(k T ; x_{h}(0), u_{2}(t)\right)\right\|_{X_{h}} \leq \delta\left\|x_{h}(0)\right\|_{X_{h}}
$$

Proof of Fact a: It follows that

$$
\begin{aligned}
& \left\|e^{\left(A_{h}+B_{h} K_{h, \Delta t}\right) T}-e^{A_{h} T}-\int_{0}^{T} e^{A_{h} \tau} d \tau B_{h} K_{h, \Delta t}\right\|_{X_{h}} \\
\leq & \left\|e^{A_{h} T}\left(e^{B_{h} K_{h, \Delta t} T}-I_{X_{h}}-B_{h} K_{h, \Delta t} T\right)\right\|_{X_{h}}+\left\|\left(e^{A_{h} T}-I_{X_{h}}\right) B_{h} K_{h, \Delta t} T\right\|_{X_{h}} \\
& +\left\|\int_{0}^{T}\left(e^{A_{h} \tau}-I_{X_{h}}\right) d \tau B_{h} K_{h, \Delta t}\right\|_{X_{h}} .
\end{aligned}
$$


Applying Proposition 4.1, it follows that there exists $\rho_{p, 2}(\cdot) \in \mathcal{K}_{\infty}$ such that

$$
\left\|e^{\left(A_{h}+B_{h} K_{h, \Delta t}\right) T}-e^{A_{h} T}-\int_{0}^{T} e^{A_{h} \tau} d \tau B_{h} K_{h, \Delta t}\right\|_{X_{h}} \leq \rho_{p, 2}(T) T,
$$

which concludes the proof of Fact 4.

Using Corollary 4.4 as well as Proposition 4.3, the proof of Corollary 4.5 is complete.

Remark 12. Corollaries 4.4 and 4.5 show that there exists positive constants $h_{p, 2}^{*}, T_{p, 2}^{*}, \alpha_{1}$ and $\alpha_{2}$ such that, for $T \in\left(0, T_{p, 2}^{*}\right)$ and $h \in\left(0, h_{p, 2}^{*}\right)$, there exists $\Delta t_{p, 2}^{*}(h)=\Delta t_{p, 1}^{*}(h)>0$ (from Corollary 4.4) such that, for $\Delta t \in\left(0, \Delta t_{p, 2}^{*}(h)\right)$, the following inequalities hold

$$
\begin{aligned}
&\left\|x_{h}\left(t ; x_{h}(0), u_{1}(t)\right)-x_{h}\left(t ; x_{h}(0), u_{2}(t)\right)\right\|_{X_{h}} \leq \alpha_{1}\left\|x_{h}(0)\right\|_{X_{h}}, \forall t \geq 0 \\
&\left\|x_{h}\left(j \Delta t ; x_{h}(0), u_{1}(t)\right)-x_{h}^{a}\left(j ; x_{h}(0)\right)\right\|_{X_{h}} \leq \alpha_{2}\left\|x_{h}(0)\right\|_{X_{h}}, \quad \forall j \in \mathbb{N}
\end{aligned}
$$

With the help of above propositions and corollaries, the next step consists of proving Theorem 3.3.

4.2. The proof of Theorem 3.3. We first re-write (2.15) as

$$
u_{T, h, \Delta t}\left(k ; x_{0}\right)=\bar{P}_{h} K_{h, \Delta t} \tilde{S}_{h, \Delta t}(k) R_{h} x_{0} .
$$

It leads to the sampled-data system (2.16)

$$
\begin{aligned}
\dot{x}(t) & \left.=A x(t)+B u_{T, h, \Delta t}\left(k ; x_{0}\right)\right) \\
& =A x(t)+B \bar{P}_{h} K_{h, \Delta t} \tilde{S}_{h, \Delta t}(k)\left(R_{h} x_{0}\right), \quad x(0)=x_{0},
\end{aligned}
$$

where $\tilde{S}_{h, \Delta t}(t)$ represents a family of semigroups that are generated from Assumption 3.

Let $\epsilon=\epsilon(\Delta, \nu)=\frac{\nu}{\left(b_{R}+b_{F}\right) \Delta}$, where $b_{R}$ and $b_{F}$ are defined in Eq. (2.8) and Condition (e) in Assumption 4 respectively. Assume that $\left\|x_{0}\right\|_{X} \leq \Delta$, and $x_{0} \in$ $\mathcal{A}_{\Delta, \nu}=\operatorname{Ker}\left(\mathcal{F}(\epsilon)-I_{X}\right)$. Let $x=P_{E} x^{E}+P_{F} x^{F}$ where $x^{E}=R_{E} x$ and $x^{F}=$ $\left(I_{X}-P_{E} R_{E}\right) x$. Let $\zeta_{0}=R_{h} x_{0}, \zeta_{0, E}=R_{h} P_{E} R_{E} x_{0}$ and $\zeta_{0, F}=R_{h}\left\{I_{X}-P_{E} R_{E}\right\} x_{0}$. For the closed-loop infinite-dimensional system (4.14), we decompose its solution along $E$ and $F$ respectively, that is,

$$
\begin{aligned}
& \dot{x}^{E}=A_{E} x^{E}+R_{E} B \bar{P}_{h} K_{h, \Delta t} \tilde{S}_{h, \Delta t}(k) \zeta_{0, E}+R_{E} B \bar{P}_{h} K_{h, \Delta t} \tilde{S}_{h, \Delta t}(k) \zeta_{0, F}, \\
& \dot{x}^{F}=A_{F} x^{F}+R_{F}\left(I_{X}-P_{E} R_{E}\right) B \bar{P}_{h} K_{h, \Delta t} \tilde{S}_{h, \Delta t}(k) \zeta_{0},
\end{aligned}
$$

where $A_{E}$ and $A_{F}$ in (4.15) and (4.16) are induced operators of $A$ in $E$ and $F$ with $C_{0}$-semigroups $S_{E}(t)$ and $S_{F}(T)$ respectively.

The proof consists of three steps.

Step 1 We first show that solutions of System (4.16) can be made sufficiently small. Proof of Step 1: The solution of System (4.16) can be written as

$$
\begin{aligned}
& x^{F}(t)=S_{F}(t) x_{F, 0}+\int_{0}^{t} S_{F}(t-s) R_{F}\left(I_{X}-P_{E} R_{E}\right) B \bar{P}_{h} K_{h, \Delta t} \tilde{S}_{h, \Delta t}(k) \zeta_{0},(4 \\
& \text { for } t \in[k T,(k+1) T), k \in \mathbb{N} .
\end{aligned}
$$


Noticing that $\tilde{S}_{h, \Delta t}(k) \zeta_{0}$ represents a family of solutions of the finite-dimensional discrete-time approximations (2.12), there exists positive constants $M_{u}, \lambda_{u}$ such that the following inequalities hold:

$$
\begin{aligned}
\left\|\tilde{S}_{h, \Delta t}(k) \zeta_{0}\right\|_{X_{h}} & \leq M_{a} e^{-\lambda_{a} T}\left\|\zeta_{0}\right\|_{X_{h}} \\
\left\|\bar{P}_{h} K_{h, \Delta t} \tilde{S}_{h, \Delta t}(k) \zeta_{0}\right\|_{X_{h}} & \leq M_{u} e^{-\lambda_{u} t}\left\|\zeta_{0}\right\|_{X_{h}},
\end{aligned}
$$

since $K_{h, \Delta t}$ is uniformly bounded, $\Delta t \in\left(0, \Delta t_{1}^{*}(h)\right)$ and $\bar{P}_{h}$ is a bounded linear operator uniformly in $h$.

By using Condition (e) in Assumption 4, it yields

$$
\begin{aligned}
\left\|S_{F}(t) x_{F, 0}\right\|_{F} & =\left\|P_{F} S_{F}(t) x_{F, 0}\right\|_{X} \leq b_{F} \epsilon\left\|x_{0}\right\|_{X} \leq b_{F} \epsilon\left\|x_{0}\right\|_{X} \\
& \leq b_{F} \epsilon \Delta,
\end{aligned}
$$

for any $x_{0} \in \bar{B}_{\Delta} \cap \operatorname{Ker}\left(\mathcal{F}_{\epsilon}-I_{X}\right)$.

On the other hand, using Assumption "HFFP" property (cf. Assumption 6), one has

$$
\begin{aligned}
& \left\|\int_{0}^{t} S_{F}(t-s) R_{F}\left(I_{X}-P_{E} R_{E}\right) B \bar{P}_{h} K_{h, \Delta t} \tilde{S}_{h, \Delta t}(k) \zeta_{0} d s\right\|_{X} \\
\leq & \epsilon b_{R}\left\|x_{0}\right\|_{X} \leq b_{R} \Delta \epsilon .
\end{aligned}
$$

By combining (4.18) with (4.19), Step 1 holds true with $\left(b_{R}+b_{F}\right) \Delta \epsilon=\nu$. 。

Step 2 We show that the solution of System (4.15) is practically exponentially stable in $h$.

Proof of Step 2: By adding and subtracting the following terms,

$$
\begin{aligned}
& R_{E} P_{h} A_{h} R_{h} P_{E} x^{E}(t), \\
& R_{E} P_{h} B_{h} K_{h, \Delta t} R_{h} P_{E} x^{E}\left(t ; P_{E} R_{E} x_{0}\right), \\
& R_{E} P_{h} B_{h} K_{h, \Delta t} R_{h} P_{E} x^{E}\left(k ; P_{E} R_{E} x_{0}\right), \\
& R_{E} B \bar{P}_{h} K_{h, \Delta t} R_{h} P_{E} x^{E}\left(k ; P_{E} R_{E} x_{0}\right), \\
& R_{E} P_{h} B_{h} K_{h, \Delta t} R_{h} P_{E} x^{E}\left(k ; P_{E} R_{E} x_{0}\right), \\
& \quad R_{E} B \bar{P}_{h} K_{h, \Delta t} x_{h}\left(k ; \zeta_{0, E}, u_{2}(t)\right), \\
& \quad R_{E} B \bar{P}_{h} K_{h, \Delta t} x_{h}\left(k ; \zeta_{0, E}, u_{1}(t)\right),
\end{aligned}
$$

for any $t \in[k T,(k+1) T)$, System (4.15) can be re-written as

$$
\begin{aligned}
& \dot{x}^{E}(t)=R_{E} P_{h} A_{h} R_{h} P_{E} x^{E}(t)+R_{E} P_{h} B_{h} K_{h, \Delta t} R_{h} P_{E} x^{E}\left(k T ; P_{E} R_{E} x_{0}\right) \\
& +R_{E}\left(A-P_{h} A_{h} R_{h}\right) P_{E} x^{E}(t) \\
& +R_{E}\left[B \bar{P}_{h}-P_{h} B_{h}\right] K_{h, \Delta t} R_{h} P_{E} x^{E}\left(t ; P_{E} R_{E} x_{0}\right) \\
& +R_{E}\left[B \bar{P}_{h}-P_{h} B_{h}\right] K_{h, \Delta t} R_{h} P_{E} R_{E}\left(x^{E}\left(k ; P_{E} R_{E} x_{0}\right)-x^{E}\left(t ; P_{E} R_{E} x_{0}\right)\right) \\
& +R_{E} B \bar{P}_{h} K_{h, \Delta t}\left[x_{h}\left(k ; \zeta_{0, E}, u_{2}(k)\right)-R_{h} P_{E} x^{E}\left(k ; P_{E} R_{E} x_{0}\right)\right] \\
& +R_{E} B \bar{P}_{h} K_{h, \Delta t}\left[x_{h}\left(k ; \zeta_{0, E}, u_{1}(t)\right)-x_{h}\left(k ; \zeta_{0, E}, u_{2}(k)\right)\right] \\
& +R_{E} B \bar{P}_{h} K_{h, \Delta t}\left[x_{h}^{a}\left(k ; \zeta_{0, E}\right)-x_{h}\left(k ; \zeta_{0, E}, u_{1}(t)\right)\right] \\
& +R_{E} B K_{h, \Delta t} x_{h}^{a}\left(k T ; \zeta_{0, F}\right) \\
& =R_{E} P_{h} A_{h} R_{h} P_{E} x^{E}(t)+R_{E} P_{h} B_{h} K_{h, \Delta t} R_{h} P_{E} x^{E}\left(k T ; P_{E} R_{E} x_{0}\right) \\
& +\varsigma_{1, h} x^{E}(t)+\varsigma_{2, h, \Delta t} x^{E}(t)+\varsigma_{3, T, h, \Delta t}(t)+\varsigma_{4, T, h, \Delta t}(t)+\varsigma_{5, T, h, \Delta t}(t) \\
& +\varsigma_{6, T, h, \Delta t}(t)+\varsigma_{7, T, h, \Delta t}(t),
\end{aligned}
$$


where

$$
\begin{aligned}
& \varsigma_{1, h} \triangleq R_{E}\left(A-P_{h} A_{h} R_{h}\right) P_{E} R_{E}, \\
& \varsigma_{2, h, \Delta t} \triangleq R_{E}\left[B \bar{P}_{h}-P_{h} B_{h}\right] K_{h, \Delta t} R_{h} P_{E} R_{E}, \\
& \varsigma_{3, T, h, \Delta t}(t) \triangleq \varsigma_{2, T, h, \Delta t}\left(x^{E}\left(k ; P_{E} R_{E} x_{0}\right)-x^{E}\left(t ; P_{E} R_{E} x_{0}\right)\right), \\
& \varsigma_{4, T, h, \Delta t}(t) \triangleq R_{E} B \bar{P}_{h} K_{h, \Delta t}\left[x_{h}\left(k ; \zeta_{0, E}, u_{2}(k)\right)-R_{h} P_{E} x^{E}\left(k ; P_{E} R_{E} x_{0}\right)\right] \\
& \varsigma_{5, T, h, \Delta t}(t) \triangleq R_{E} B \bar{P}_{h} K_{h, \Delta t}\left[x_{h}\left(k ; \zeta_{0, E}, u_{1}(t)\right)-x_{h}\left(k ; \zeta_{0, E}, u_{2}(k)\right)\right], \\
& \varsigma_{6, T, h, \Delta t}(t) \triangleq R_{E} B \bar{P}_{h} K_{h, \Delta t}\left[x_{h}^{a}\left(k ; \zeta_{0, E}\right)-x_{h}\left(k ; \zeta_{0, E}, u_{1}(t)\right)\right], \\
& \varsigma_{7, T, h, \Delta t}(t) \triangleq R_{E} B \bar{P}_{h} K_{h, \Delta t} x_{h}^{a}\left(k ; \zeta_{0, F}\right) .
\end{aligned}
$$

Then, the solution of System (4.15) becomes

$$
\begin{aligned}
x^{E}(t)= & S_{T, h}^{E}(t) x^{E}(0)+\int_{0}^{t} S_{T, h}^{E}(t-s) \varsigma_{1, h} x^{E}(s) d s \\
& +\int_{0}^{t} S_{T, h}^{E}(t-s) \varsigma_{2, h, \Delta t} x^{E}(s) d s+\int_{0}^{t} S_{T, h}^{E}(t-s) \varsigma_{3, T, h, \Delta t}(s) d s \\
& +\int_{0}^{t} S_{T, h}^{E}(t-s) \varsigma_{4, T, h, \Delta t}(s) d s+\int_{0}^{t} S_{T, h}^{E}(t-s) \varsigma_{5, T, h, \Delta t}(s) d s \\
& +\int_{0}^{t} S_{T, h}^{E}(t-s) \varsigma_{6, T, h, \Delta t}(s) d s \\
& +\int_{0}^{t} S_{T, h}^{E}(t-s) \varsigma_{7, T, h, \Delta t}(s) d s .
\end{aligned}
$$

The proof will be completed by showing the following facts.

Fact 1 Let $\rho_{a}(\cdot)$ be a class $\mathcal{K}_{\infty}$-function. Then, there exists $h_{a}>0$ such that, for $h \in\left(0, h_{a}\right)$, the following inequality holds

$$
\left\|R_{E}\left(A-P_{h} A_{h} R_{h}\right) P_{E}\right\|_{E} \leq \rho_{a}(h) .
$$

Proof of Fact 1: Note that $R_{E} A P_{E}$ is an endomorphism of $E$ thus it is bounded as well as $R_{E} P_{h} A_{h} R_{h} P_{E}$. Using Assumption 1, it yields

$$
\left\|R_{E} A P_{E}-R_{E} P_{h} A_{h} R_{h} P_{E}\right\|_{E} \underset{h \rightarrow 0}{\longrightarrow} 0 .
$$

Therefore Fact 1 holds true.

Using Fact 1 , let $\delta>0$ be arbitrary. One has

$$
\begin{aligned}
\left\|\int_{0}^{t} S_{T, h}^{E}(t-s) \varsigma_{1, h} x^{E}(s) d s\right\|_{E} & \leq \int_{0}^{t} M_{E} e^{-\lambda_{E}(t-s)} \rho_{a}(h)\left\|x^{E}(s)\right\|_{E} d s \\
& \leq \int_{0}^{t} \frac{\delta}{2}\left\|x^{E}(s)\right\|_{E} d s,
\end{aligned}
$$

by choosing $h$ small enough.

Fact 2 Let $\rho_{b}(\cdot)$ be a class $\mathcal{K}_{\infty}$-function. There exists $h_{b}>0$ such that, for $h \in\left(0, h_{b}\right)$, the following inequality holds

$$
\left\|R_{E}\left(B \bar{P}_{h}-P_{h} B_{h}\right) K_{h, \Delta t} R_{h} P_{E}\right\|_{E} \leq \rho_{b}(h) .
$$

Proof of Fact 2: Similar to the proof of Fact 1, using the fact that $E$ is finite-dimensional space, the result holds. 
Using Fact 2, let $\delta>0$ be arbitrary, we have

$$
\begin{aligned}
\left\|\int_{0}^{t} S_{T, h}^{E}(t-s) \varsigma_{2, h, \Delta t} x^{E}(s) d s\right\|_{E} & \leq \int_{0}^{t} M_{E} e^{-\lambda_{E}(t-s)} \rho_{b}(h)\left\|x^{E}(s)\right\|_{E} d s \\
& \leq \int_{0}^{t} \frac{\delta}{2}\left\|x^{E}(s)\right\|_{E} d s
\end{aligned}
$$

by choosing $h$ small enough.

Fact 3 For any $\delta>0$, there exists $T_{2}^{*}>0$ such that the following inequality holds

$$
\left\|x^{E}\left(k ; P_{E} R_{E} x_{0}\right)-x^{E}\left(t ; P_{E} R_{E} x_{0}\right)\right\|_{E} \leq \delta
$$

Fact 4 For any fixed $t>0$, there exists a class $\mathcal{K}_{\infty}$-function $\rho_{c}(\cdot)$ such that, for $z_{0} \in E$, the following holds.

$$
\left\|R_{E} P_{h}\left\{x_{h}\left(s ; R_{h} P_{E} z_{0}, u_{2}(k)\right)-R_{h} P_{E} R_{E} z\left(s ; z_{0}\right)\right\}\right\|_{E} \leq \rho_{c}(h),(4.31)
$$

for all $s \in[0, t]$. Here $z\left(t ; z_{0}\right)$ represents solutions of System (3.5) with the initial condition $z_{0}$.

Proof of Fact 4: Note that $x_{T, h}\left(k ; R_{h} P_{E} z_{0}\right)$ represents solutions of a System (3.4), whose initial condition is $R_{h} P_{E} z_{0}$ and $u_{2}(t)=K_{h, \Delta t} x(k T), \forall t \in$ $[k T,(k+1) T), k \in \mathbb{N}$.

Denoting $\xi_{h}(t)=R_{h} P_{E} z(t)$, we have two systems in $X_{h}$,

$$
\begin{aligned}
& \dot{x}_{h}(t)=A_{h} x_{h}(t)+B_{h} K_{h, \Delta t} x_{h}(k T), x_{h}(0)=R_{h} P_{E} z_{0}, \\
& \dot{\xi}_{h}(t)=R_{h} P_{E} R_{E} P_{h}\left[A_{h} \xi_{h}(t)+B_{h} K_{h, \Delta t} \xi_{h}(k T)\right], \xi_{h}(0)=R_{h} P_{E} z_{0} .
\end{aligned}
$$

When they are projected onto $E$, it follows that

$$
\begin{aligned}
R_{E} P_{h} \dot{x}_{h}(t) & =R_{E} P_{h}\left[A_{h} x_{h}(t)+B_{h} K_{h, \Delta t} x_{h}(k T)\right], \\
R_{E} P_{h} \dot{\xi}_{h}(t) & =R_{E} P_{h}\left\{R_{h} P_{E} R_{E} P_{h}\left[A_{h} \xi_{h}(t)+B_{h} K_{h, \Delta t} \xi_{h}(k T)\right]\right\} \\
& =R_{E} P_{h} R_{h}\left(P_{E} R_{E} P_{h}\right)\left[A_{h} \xi_{h}(t)+B_{h} K_{h, \Delta t} \xi_{h}(k T)\right] .
\end{aligned}
$$

Obviously

$$
\left\|R_{E} P_{h} R_{h} P_{E}-I_{E}\right\|_{\mathcal{L}(E)} \underset{h \rightarrow 0}{\longrightarrow} 0
$$

in which the convergence is uniform for small $h$ since $\operatorname{dim} E<\infty$. Note that $P_{h}$ is uniformly bounded and $P_{E}$ and $R_{E}$ are bounded according to the Lax-Richtmyer Equivalent Theorem [25]. ${ }^{34}$.

Fact 5 There exist positive constant $\alpha_{3}$ and $h_{2}^{*}$ such that, for $h \in\left(0, h_{2}^{*}\right), t \in$ $(0, \infty)$ and $z_{0} \in E$, one has

$$
\left\|R_{E} P_{h}\left\{x_{h}\left(t ; R_{h} P_{E} z_{0}, u_{2}(k)\right)-R_{h} P_{E} R_{E} z\left(t ; z_{0}\right)\right\}\right\|_{E} \leq \alpha_{3}\left\|z_{0}\right\|_{E} \cdot(4
$$

\footnotetext{
${ }^{3}$ Since $A_{h} \triangleq R_{h} A P_{h} \in X_{h}$, and by using Fact 1 in the proof of Corollary $4.5, e^{R_{h} A P_{h} t}$ is uniformly bounded for any finite time $t$. Therefore Lax-Richtmyer Equivalent Theorem is applicable.

${ }^{4}$ It is worthwhile to note that, since two solutions are evaluated in $E$, the convergence does not depends on the choice of $z_{0}$. In general, $\rho_{c}(\cdot)$ is determined by the given initial condition when an infinite-dimensional space $X$ is considered.
} 
Proof of Fact 5: Given $t>0$ and $z_{0}$, then choosing $h_{2}^{*}=\rho_{c}^{-1}\left(\frac{\delta}{2}\right)$ and using Fact 4 yield

$$
\left\|R_{E} P_{h}\left\{x_{h}\left(s ; R_{h} P_{E} z_{0}, u_{2}(k)\right)-R_{h} P_{E} R_{E} z\left(s ; z_{0}\right)\right\}\right\|_{E} \leq \delta, \forall s \in[0, t] .
$$

Assumption 8 implies that $R_{E} P_{h} R_{h} P_{E} R_{E} z\left(s ; z_{0}\right)$ is exponentially stable, uniformly in small $h$. Using Proposition 4.3 and Assumption 8, it can be shown that $R_{E} P_{h} x_{h}\left(s ; R_{h} P_{E} z_{0}, u_{2}(k)\right)$ is exponentially stable, uniformly for small $h$. This concludes the proof of Fact 5 .

Fact $6 R_{E} B K_{h, \Delta t}$ is uniformly bounded by $B_{E, T, h}$ for $T>0, k \in \mathbb{N}, h \in\left(0, h^{*}\right)$ and $\Delta t \in\left(0, \Delta t^{*}(h)\right.$, where $h^{*}$ and $\Delta t^{*}(h)$ are defined in Assumption 7. Proof of Fact 6: Note that $R_{E} B$ is a compact operator as $R_{E} B$ has a finite range. Using Banach-Steinhaus Theorem [21, page 249] yields the result.

We finally prove the theorem as follows. From what precedes, Facts 1 to 3 handle $\varsigma_{1, T, h, \Delta t}(t)$ to $\varsigma_{3, T, h, \Delta t}(t)$ respectively. Moreover, Fact 4 and 5 handle $\varsigma_{4, T, h, \Delta t}(t)$ and Fact 6 finally handles $\varsigma_{7, T, h, \Delta t}(t)$.

Moreover, Corollaries 4.4 and 4.5 take care of $\varsigma_{5, T, h, \Delta t}(t)$ and $\varsigma_{6, T, h, \Delta t}(t)$ respectively. By combining Facts 1-6 with the two corollaries and by using Gronwall Lemma, it yields

$$
\begin{aligned}
\left\|x^{E}(t)\right\|_{E} \leq & \left\|S_{T, h}^{E}(t)\right\|_{E}\left\|P_{E} R_{E} x_{0}\right\|_{E}+\int_{0}^{t}\left\|S_{T, h}^{E}(t-s)\right\|_{E} \rho_{a}(h)_{E}\left\|x^{E}(s)\right\|_{E} d s \\
& +\int_{0}^{t}\left\|S_{T, h}^{E}(t-s)\right\|_{E} \rho_{b}(h)\left\|x^{E}(s)\right\|_{E} d s \\
& +\int_{0}^{t}\left\|S_{T, h}^{E}(t-s)\right\|_{E} \rho_{b}(h) \delta d s \\
& +\int_{0}^{t}\left\|S_{T, h}^{E}(t-s)\right\|_{E} \alpha_{3}\left\|P_{E} R_{E} x_{0}\right\|_{E} d s \\
& +\int_{0}^{t}\left\|S_{T, h}^{E}(t-s)\right\|_{E} \alpha_{1}\left\|R_{h} P_{E} R_{E} x_{0}\right\|_{X_{h}} d s \\
& +\int_{0}^{t}\left\|S_{T, h}^{E}(t-s)\right\|_{E} \alpha_{2}\left\|R_{h} P_{E} R_{E} x_{0}\right\|_{X_{h}} d s \\
& +\int_{0}^{t} S_{T, h}^{E}(t-s) B_{E, T, h} M_{a}\left\|R_{h} P_{E} R_{E} x_{0}\right\|_{X_{h}} d s . \\
\leq & M e^{-\lambda t}\left\|x_{0}\right\|_{X} .
\end{aligned}
$$

Since $\left\|x^{E}\right\|_{E}=\left\|P_{E} x^{E}\right\|_{X}$, the proof of Theorem 3.3 is now completed by combining Step 1 and Step 2 .

5. Conclusions. In this paper, practical exponential stability properties of the sampled-data infinite-dimensional systems using controllers generated from numerical approximations are discussed. The controllers are first designed such that they uniformly exponentially stabilize a family of finite-dimensional discrete-time approximations. Then, they are used in sampled-data infinite-dimensional systems. Under some tight sufficient conditions, by tuning the parameters $(T, h, \Delta t)$, the resulting controllers will practically stabilize the sampled-data infinite-dimensional system for any initial condition $x_{0} \in B_{\Delta}$ that is properly filtered. 


\section{REFERENCES}

[1] H.T. BANKS AND K. ITO, Approximation in LQR problems for infinite dimensional systems with unbounded input operators, J. Math. Systems Estim. Control 7 (1997), pp. 1-34.

[2] H.T. Banks, K. Ito AND Y. Wang, Well posedness for damped second order systems with unbounded input operators, Differential and Integral Equations, 8 (1995), pp. 587-606.

[3] H.T. BANKS AND K. Kunisch, The linear regulator problem for parabolic systems, SIAM J. Control Optim. 22 (1984), pp. 684-698.

[4] R. Banning, W. L DE Koning, Spectral analysis of stochastically sampled dynamic systems, Math. Control Signals Syst. 14 (2001), pp. 86-107.

[5] T. Chen and B.Francis, Optimal Sampled-data Control Systems, (1995), Springer-Verlag: London.

[6] F. H. Clarke, Y.S. Ledyaev, E.D. Sontag, and A.I. Subbotin, Asymptotic controllability implies feedback stabilization. IEEE Trans. Automat. Control, 42 (1997), pp. 1394-1407.

[7] J.M. Coron and E. TrÉlat, Global steady-state controllability of 1-d semilinear heat equations. SIAM J. Cont. Optim. 43 (2004), pp. 549-569.

[8] J.M. Coron and E. TrÉLAT, Global steady-state stabilization and controllability of 1-d semilinear wave equations. Commun. Contemp. Math., 8(2006), pp. 535-567.

[9] J. Coughlan and H. Logemann, Absolute stability and integral control for infinitedimensional discrete-time systems, Nonlinear Analysis, 71 (2009), pp. 4769-4789.

[10] R. Courant And K. Friedrichs and H. Lewy, Ü ber die partiellen Differenzengleichungen der mathematischen Physik, Math. Ann. 100 (1928), pp. 32-74.

[11] R.F. Curtain and H.J. Zwart, An Introduction To Infinite-Dimensional Linear Systems Theory, (1995), Springer-Verlag: New York.

[12] S. ERvedoza ANd E. Zuazua, Uniformly exponentially stable approximations for a class of damped systems, Journal de Mathématiques Pures et Appliquées, 91 (2009), pp. 20-48.

[13] J.S. GIBSON, The riccati integral equations for optimal control problems on Hilbert spaces, SIAM J. Control Optim. 27 (1979), pp. 537-565.

[14] J. S. Gibson AND I. G. Rosen, Numerical approximation for the infinite-dimensional discretetime optimal linear-quadratic regulator problem, SIAM Journal on Control and Optimization, 26, (1988), pp. 428-451.

[15] C.A. Hall and T.A. Prosching, Numerical Analysis of Partial Differential Equations, (1990), Prentice Hall, Englewood Cliffs, New Jersy.

[16] S. HidekI, Sampled-data $H_{\infty}$ control of linear parabolic systems with unbounded output operators, Transactions of the Society of Instrument and Control Engineers, 40, (2004), pp. 915-924.

[17] F. Kappel, F. and D.Salamon, An approximation theorem for the algebraic riccati equation. SIAM J. Control Optim. 28 (1990), pp. 1136-1147.

[18] Z. Ke, H. Logemann And R. Rebarber, A sampled-data servomechanism for stable well-posed systems, IEEE Trans. Automatic Control, 54, (2009), pp. 1123-1128.

[19] Z. Ke, H. Logemann And S. Townley, Adaptive sampled-data integral control of stable infinite-dimensional linear systems, Systems and Control Letters, 58 (2009), pp. 233-240.

[20] K. Komornik, Exact Controllability and Stabilizations, the Multiplier Method, (1994), Wiley, Masson, Paris.

[21] E. Kreyszig, Introductory Function Analysis with Applications, (1989), John Wiley \& Sons.

[22] S. LABBÉ AND E. TRÉLAT, Uniform controllability of semidiscrete approximations of parabolic control systems, Systems and Control Letters 55 (2006), pp. 597-609.

[23] I. Lasiecka and R. Triggiani, Stabilization and structural assignment of dirichlet boundary feedback parabolic euqations. SIAM J. Control and Optimiztions, 21 (1983), pp. 766-803.

[24] I. Lasiecka And R. Triggiani, Control Theory for Partial Differential Equations: Continous and Approximation Theories. I. Abstract Parabolic Systems.. Vol. 74 of Encyclopedia of Methematics and its Applications, (2000), Cambridge University Press.

[25] P.D. Lax, P.D. and R.D. Richtmyer, Survey of the stability of linear finite difference equations, Communications on Pure and Applied Mathematics, 4 (1956), pp. 267-293.

[26] J.L. LIONS, Exact controllability, stabilization and perturbations for distributed systems, SIAM Rev. 30 (1988), pp. 1-68.

[27] Z. LiU And S. Zheng, Semigroups associated with dissipative systems, (1999), Chapman \& Hall/CRC, Research Notes in Mathematics.

[28] H. Logemann and R. Rebarber and S.Townley, Stability of infinite dimensional sampleddata systems, Trans. American Mathematical Society, 35 (2003), pp. 301-328.

[29] H. Logemann, R. Rebarber and S. Townley, Generalized sampled-data stabilization of wellposed linear infinite-dimensional systems, SIAM J. Control and Optimization, 44 (2005), 
pp. $1345-1369$.

[30] D. NešIĆ AND A. R. TEel, Sampled-data control of nonlinear systems: an overview of recent results, In Perspectives in Robust Control, (Ed. R.S.O.Moheimani) (2001), Springer Verlag, pp. 259-270.

[31] D. Nešić And A. R. Teel And P. V. Кокотovic Sufficient conditions for stabilization of sampled-data nonlinear systems via discrete-time approximations, Systems and Control Letters, 38 (1999), pp. 259-270.

[32] A. Pazy. Semigroups of Linear Operator and Applications to Partial Differential Equations, (1983), Springer-Verlag: New York.

[33] K.RAMDANI AND T.TAKAHASHI AND M.TUCSNAK Uniformly exponentially stable approximations for a class of second order evolution equations. application to LQR optimization problems, ESAIM - Optimisation and Calculus of Variations 13 (2007), pp. 503-527.

[34] R. Rebarber, And S. Townley, Generalized sampled data feedback control of distributed parameter systems, Systems \& Control Letters, 34 (1998), pp. 229-240.

[35] R. Rebarber, AND S. TOWnLey, Robustness of distributed parameter systems with respect to sample and hold-counterexamples, Proceedings of the 2000 IEEE Conference on Decision and Control, (2000), Syndey, Australia.

[36] R. Rebarber and G. Weiss, Necessary conditions for exact controllability with a finitedimensional input space, Syst. Control Lett. 40 (2000), pp. 217-227.

[37] I. G. Rosen AND C. WANG, On stabilizability and sampling for infinite dimensional systems, IEEE Transactions on Automatic Control, 37, (1992), pp. 1653-1656.

[38] D.L.Russell, Controllability and stabilizability theory for linear partial differential equations: recent progress and open questions, SIAM Rev. 20 (1978), pp. 639-739.

[39] E. D. Sontag and H.J. Sussmann. Remarks on continuous feedback. In Proc. IEEE Conf. Decision and Control, Albuquerque, Dec.1980, (1980), pp. 916-921.

[40] Y. TAN AND D. NeŠIĆ AND A.R. TEEL, A trajectory based approach for robust stability properties of infinite-dimensional systems, In: Proc. $7^{\text {th }}$ IFAC Symposium on Nonlinear Control Systems, (2007), Pretoria, South Africa.

[41] L.T. TÉBOU AND E.ZuAzuA, Uniform exponential long time decay for the space semidiscretization of a locally damped wave equation via an artificial numerical viscosity, Numer. Math. 95 (2003), pp. 563-598.

[42] L.T. TÉBOU AND E. ZuAzUA, Uniform boundary stabilization of the finite difference space discretization of the 1-d wave equation, Advances in Computational Mathematics, 26 (2007), pp. 337-365.

[43] X. Zhang, C. Zheng and E. Zuazua, Time discrete wave equations: Boundary observability and control, Discrete and Continuous Dynamical Systems, 23 (2009), pp. 571-604.

[44] E. Zuazua, Propagation, observation, control and numerical approximation of waves approximated by finite difference method, SIAM Rev. 47 (2005), pp. 197-243.

[45] E. Zuazua, Control and numerical approximation of the wave and heat equations, Proceedings of Internat. Congress Math, (2006), pp. 1389-1417. 